\title{
Determining the optimal piecewise constant approximation for the nonhomogeneous Poisson process rate of Emergency Department patient arrivals
}

\author{
Alberto De Santis ${ }^{1}(1) \cdot$ Tommaso Giovannelli $^{1}\left(\mathbb{0} \cdot\right.$ Stefano Lucidi $^{1}(1)$. \\ Mauro Messedaglia ${ }^{2} \cdot$ Massimo Roma $^{1}$
}

Accepted: 17 February 2021 / Published online: 11 March 2021

(c) The Author(s) 2021

\begin{abstract}
Modeling the arrival process to an Emergency Department (ED) is the first step of all studies dealing with the patient flow within the ED. Many of them focus on the increasing phenomenon of ED overcrowding, which is afflicting hospitals all over the world. Since Discrete Event Simulation models are often adopted to assess solutions for reducing the impact of this problem, proper nonstationary processes are taken into account to reproduce time-dependent arrivals. Accordingly, an accurate estimation of the unknown arrival rate is required to guarantee the reliability of results. In this work, an integer nonlinear black-box optimization problem is solved to determine the best piecewise constant approximation of the time-varying arrival rate function, by finding the optimal partition of the $24 \mathrm{~h}$ into a suitable number of not equally spaced intervals. The black-box constraints of the optimization problem make the feasible solutions satisfy proper statistical hypotheses; these ensure the validity of the nonhomogeneous Poisson assumption about the arrival process, commonly adopted in the literature, and prevent mixing overdispersed data for model estimation. The cost function of the optimization problem includes a fit error term for the solution accuracy and a penalty term to select an adequate degree of regularity of the optimal solution. To show the effectiveness of this methodology, real data from one of the largest Italian hospital EDs are used.
\end{abstract}

Keywords Emergency Department · Arrival process · Non homogeneous Poisson process $\cdot$ Black-box optimization

Tommaso Giovannelli

giovannelli@diag.uniroma1.it

Extended author information available on the last page of the article 


\section{Introduction}

Statistical modeling for describing and predicting patient arrival to Emergency Departments (EDs) represents a basic tool of each study concerning ED patient load and crowding. Indeed, all the approaches adopted to this aim require an accurate model of the patient arrival process. Of course, such a process plays a key role in tackling the widespread phenomenon of overcrowding which afflicts EDs all over the world (see e.g., Ahalt et al. (2018), Bernstein et al. (2003), Daldoul et al. (2018), Hoot and Aronsky (2008), Hoot et al. (2007), J Reeder et al. (2003), Vanbrabant et al. (2020), Wang et al. (2015), Weiss et al. (2004), Weiss et al. (2006)). The two factors that have the most significant effect on overcrowding are both external and internal. The first concerns the patient arrival process; the second regards the patient flow within the ED. Therefore, both aspects must be accurately considered for a reliable study on ED operation.

Several modeling approaches for analyzing ED patient flow have been proposed in literature (see Wiler et al. (2011) for a survey). The main quantitative methods used are based on statistical analysis (time-series, regression) or general analytic formulas (queuing theory). In particular, a realistic model for patient arrivals is crucial for dealing with important issues regarding the patient flow through an ED. To this aim, time-dependent queueing models have been successfully adopted; for instance, in Vile et al. (2017) staffing level problem has been efficiently dealt with using queuing theoretical approach in a time-dependent setting with a time-varying input. However, simulation modeling (both Discrete Event and Agent-Based Simulation) is currently one of the most widely used and flexible tool for studying the patient flow through an ED. It enables performing effective scenario analysis, aiming at determining bottlenecks (if any) and testing different ED settings. We refer to Salmon et al. (2018) for a recent survey on simulation modeling for ED operation.

Simulation modeling can be also combined with other techniques to improve the responses provided; for instance, in the recent paper by Gartner and Padman (2020) a Discrete Event Simulation model has been linked with machine learning models for better estimating the patient perception of the services' delay provided in emergency care.

In the time-dependent modelling approach, a methodology that appears to be a step forward is Simulation-Based Optimization. It combines a simulation model with a black-box optimization algorithm, aiming at determining an optimal ED setting, based on suited objective function (representing some KPIs) to be maximized or minimized (Ahmed and Alkhamis 2009; Guo et al. 2016, 2017).

Modeling methodologies are generally based on assumptions that, in some cases, may represent serious limitations when applied to complex real-world cases, such as ED operation. In particular, when dealing with ED patient arrival stochastic modeling, due to the nonstationarity of the process, a standard assumption is the use of Nonhomogeneous Poisson Process (NHPP) (see e.g., Ahalt et al. (2018), Ahmed and Alkhamis (2009), Guo et al. (2017), Kim and Whitt (2014a), Kuo et al. (2016), Zeinali et al. (2015)). We recall that a counting process $X(t)$ is 
an NHPP if 1) arrivals occur one at a time (no batch); 2) the process has independent increments; 3 ) increments have Poisson distribution, i.e. for each interval $\left[t_{1}, t_{2}\right]$

$$
P\left(X\left(t_{1}\right)-X\left(t_{2}\right)=n\right)=e^{-m\left(t_{1}, t_{2}\right)} \frac{\left[m\left(t_{1}, t_{2}\right)\right]^{n}}{n !},
$$

where $m\left(t_{1}, t_{2}\right)=\int_{t_{1}}^{t_{2}} \lambda(s) d s$ and $\lambda(t)$ is the arrival rate. Unlike the Poisson process (where $\lambda(t)=\lambda$ ), NHPP has nonstationary increments and this makes the use of NHPP suitable for modeling ED arrival process, which is usually strongly time-varying. Of course, appropriate statistical tests must be applied to available data to check if NHPP fits. This is usually performed by assuming that NHPP has a rate that can be considered approximately piecewise constant. Hence, Kolmogorov-Smirnov (KS) statistical test can be applied in separate and equally spaced intervals, and usually the classical Conditional-Uniform (CU) property of the Poisson process is exploited (see Brown et al. (2005), Kim and Whitt (2014a), Kim and Whitt (2014b)). Unlike the standard KS test, in the CU KS test, the data are transformed before applying the test. More precisely, by CU property, the piecewise constant NHPP is transformed into a sequence of i.i.d. random variables uniformly distributed on $[0,1]$ so that it can be considered a (homogeneous) Poisson process in each interval. In this manner, the data from all the intervals can be merged into a single sequence of i.i.d. random variables uniformly distributed on $[0,1]$. This procedure, proposed in Brown et al. (2005), enables removing nuisance parameters obtaining independence from the rate of the Poisson process on each interval. Hence data from separate intervals (with different rates on each of them) and also from different days can be combined, avoiding common drawback due to large intra-day and inter-day variation of the ED patient arrival rate. Brown et al. (2005) apply the CU $\mathrm{KS}$ test after performing a further logarithmic data transformation. In Kim and Whitt (2014b), Kim and Whitt (2015), this approach has been extensively tested along with alternative data transformations proposed in early papers Durbin (1961) and Lewis (1965). However, in Kim and Whitt (2014a) the authors have observed that this procedure applied to ED patient arrival data is fair only if they are "analyzed carefully". This is because the following three issues must be seriously considered: 1) data rounding, 2) choice of the intervals, 3) overdispersion. The first issue may produce batch arrivals (zero-length interarrival times) that are not included in an NHPP, so that unrounded data (or an unrounding procedure) must be considered. The second is a major issue in dealing with ED patient arrivals since the arrival rate can rapidly change so that the piecewise constant approximation is reasonable only if the intervals are properly chosen. The third issue regards combining data from multiple days. Indeed, in studying the ED patient arrival process, it is common to combine data from the same time slot from different weekdays, being this imperative when data from a single day are not sufficient for statistical testing. Data collected from the EDs database usually show large variability over successive weeks mainly due to seasonal phenomena like flu season, holiday season, etc. However, this overdispersion phenomenon must be checked by using a dispersion test on the available data (e.g., Kathirgamatamby (1953)). 
In this work, we propose a new modeling approach for the ED patient arrival process based on a piecewise constant approximation of the arrival rate accomplished with not equally spaced intervals. This choice is suggested by the typical situation that occurs in EDs where the arrival rate is small and varying during the night hours, and it is higher and more stable in the daytime, this is indeed what happens in the chosen case study. It is worth noting that ED management typically plans resource allocation based on the average number of arrivals expected in a given time-slot (corresponding to an interval of the partition of the $24 \mathrm{~h}$ ), for instance in staffing allocation. Therefore, it is important to have fewer intervals to represent the real arrival process, still ensuring good accuracy. In this respect, to obtain an accurate representation of the arrival rate $\lambda(t)$ by a piecewise constant function $\lambda_{D}(t)$, a finer discretization of the time-domain is required during the night hours, as opposed to daytime. For this reason, the proposed method finds the best partition of the $24 \mathrm{~h}$ into intervals not necessarily equally spaced.

As far as the authors are aware, the use of an optimization method for identifying stochastic processes characterizing the patient flow through an ED was already proposed in Guo et al. (2016), but that study aimed at determining the optimal service time distribution parameters (by using a metaheuristic approach) and it did not involve ED arrival process. Therefore our approach represents the first attempt to adopt an optimization method for determining the best stochastic model for the ED arrival process. In the previous work De Santis et al. (2020) a preliminary study was performed following the same approach. Here, concerning De Santis et al. (2020), we propose a significantly enhanced statistical model which allows us to obtain better results on the case study we consider.

In constructing a statistical model of the ED patient arrivals, a natural way to define a selection criterion is to evaluate the fit error between $\lambda(t)$ and its approximation $\lambda_{D}(t)$. However, the true arrival rate is unknown. In the approach we propose, as opposed to Kim and Whitt (2014a), no analytical model is assumed for $\lambda(t)$, but it is substituted by an "empirical arrival rate model" $\lambda_{F}(t)$ obtained by a sample approximation corresponding to the very fine uniform partition of the $24 \mathrm{~h}$ into intervals of 15 minutes. In each of these intervals, the average arrival rate values have been estimated from data obtained by collecting samples over the same day of the week, for all the weeks in some months, using experimental data for the ED patient arrival times. Hence, any other $\lambda_{D}(t)$ corresponding to a grosser partition of the day must be compared to $\lambda_{F}(t)$. In other words, an optimization problem is solved to select the best day partition in not equally spaced intervals, determining a piecewise constant approximation of the arrival rate over the $24 \mathrm{~h}$ with the best fit to the empirical model. Therefore, the objective function (to be minimized) of the optimization problem we formulate, comprises the fit error, namely the mean squared error. Moreover, an additional penalty term is included aiming at obtaining the overall regularity of the optimal approximation, being the latter measured using the sum of the squares of the jumps between the values in adjacent intervals. The rationale behind this term is to avoid optimal solutions with too rough behavior, namely few long intervals with high jumps. 
To make the result reliable, several constraints must be considered. First, the length of each interval of the partition can not be less than a fixed value (half an hour, $1 \mathrm{~h}$ ). Moreover, for each interval,

- the CU KS test must be satisfied to support the NHPP hypothesis;

- the dispersion test must be satisfied to ensure that data are not overdispersed, and could be considered as a realization of the same process (no week seasonal effects).

The resulting problem is a black-box constrained optimization problem and to solve it we use a method belonging to the class of Derivative-Free Optimization. In particular, we use the new algorithmic framework recently proposed in Liuzzi et al. (2020) which handles black-box problems with integer variables.

We performed extensive experimentation on data collected from the ED of a big hospital in Rome (Italy), also including some significant sensitivity analyses. The results obtained show that this approach enables determining the number of intervals and their length such that an accurate approximation of the empirical arrival rate is achieved, ensuring the consistency between the NHPP hypothesis and the arrivals data. The regularity of optimal piecewise constant approximation can be also finely tuned by properly weighing the penalty term in the objective function concerning the fit error term.

It is worth noting that the use of a piecewise constant function for approximating the arrival rate function is usually required by the most common discrete event simulation software packages when implementing the ED patient arrivals process as an NHPP.

To summarize, we propose a model for the patient arrival process at an ED under NHPP hypotheses, aiming at defining the best piecewise constant approximation of arrival rate with not equally spaced intervals. This is obtained by solving an integer nonlinear black-box optimization problem where the number of intervals is not a priori fixed and the constraints ensure that the solution complies with the NHPP hypothesis.

The paper is organized as follows. In Sect. 2 we describe the statistical model we propose along with the optimization problem we consider. Sect. 3, briefly reports information on the hospital ED under study. The results of extensive experimentation are included in Sect. 4, while Sect. 5 reports a preliminary assessment of the approach we propose. Finally, Sect. 6 includes some concluding remarks.

\section{Analytical model}

The arrival process at EDs is usually characterized by a strong intra-day variation both in the arrival rate and interarrival times: typically, experimental data show rapid changes in the number of arrivals during the night hours, as opposed to a smoother profile at daytime. As we already mentioned in the Introduction, for this reason, the ED arrival process is usually modeled as an NHPP. 


\subsection{Statistical model}

We describe the statistical model of the ED patient arrivals we propose. As opposed to Kim and Whitt (2014a), we do not assume any analytical model for the arrival rate $\lambda(t)$, and therefore a suitable representation of the unknown function is needed. A realistic representation can be obtained by averaging the number of arrivals observed in experimental data on suitable intervals over the $24 \mathrm{~h}$ of the day, not necessarily equally spaced.

Let $\left\{T_{i}\right\}$ denote a partition $P$ of the observation period $T=[0,24]$ (h) in $N$ intervals, and let $\left\{\lambda_{i}\right\}$ be the corresponding sample average rates. Then a piecewise constant approximation of $\lambda(t)$ can be written

$$
\lambda_{D}(t)=\sum_{i=1}^{N} \lambda_{i} \mathbf{1}_{T_{i}}(t), \quad t \in T,
$$

where $\mathbf{1}_{T_{i}}(t)$ is 1 for $t \in T_{i}$ and 0 otherwise (the indicator function of set $T_{i}$ ). Any partition $P$ gives rise to a different approximation $\lambda_{D}(t)$, depending on the number of intervals and their lengths. Therefore a criterion is needed to select the best partition $P^{\star}$ with some desirable features.

First of all, we need to ensure that there is no overdispersion in the arrivals data. We refer to the commonly used dispersion test proposed in Kathirgamatamby (1953) and reported in Kim and Whitt (2014a). If it is satisfied, then it is possible to combine arrivals for the same day of the week over different weeks. To this aim, for any partition $P$, let $\left\{k_{i}^{r}\right\}$ denote the number of arrivals in the $i$-th partition interval $T_{i}$ in the $r$-th week, $r=1, \ldots, m$. Consider the statistics

$$
D s_{i}=\frac{1}{\mu_{i}} \sum_{r=1}^{m}\left(k_{i}^{r}-\mu_{i}\right)^{2}, \quad i=1, \ldots, N,
$$

where $\mu_{i}=\frac{1}{m} \sum_{r=1}^{m} k_{i}^{r}$ is the average number of arrivals in the given interval for the same day of the week over the considered $m$ weeks. Under the null hypothesis that the counts $\left\{k_{i}^{r}\right\}$ are a sample of $m$ independent Poisson random variables with the same mean count $\mu_{i}$ (no overdispersion), then $D s_{i}$ is distributed as $\chi_{m-1}^{2}$, the chisquared distribution with $m-1$ degrees of freedom. Therefore the null hypothesis is not rejected with $1-\alpha$ confidence level if

$$
D s_{i} \leq \chi_{m-1, \alpha}^{2}, \quad i=1, \ldots, N
$$

where $\chi_{m-1, \alpha}^{2}$ is, of course, the $\alpha$ level critical value of the $\chi_{m-1}^{2}$ distribution.

Furthermore, the partition is feasible if data are consistent with NHPP. Namely, if we denote by $k_{i}$ the number of arrivals in each interval $T_{i}=\left[a_{i}, b_{i}\right)$ obtained by considering data of the same weekday, in the same interval, over $m$ weeks, i.e. $k_{i}=\sum_{r=1}^{m} k_{i}^{r}$, $i=1, \ldots, N$, the partition is feasible if each $k_{i}$ has a Poisson distribution with a rate $\lambda_{i}$ obtained as $\mu_{i} /\left(b_{i}-a_{i}\right)$. To check the validity of the Poisson hypothesis, the CU KS test can be performed (see Brown et al. (2005), Kim and Whitt (2014a)). We prefer to use CU KS rather than the Lewis KS test since this latter is highly sensitive to rounding 
of the numerical values and the CU KS test has more power against alternative hypotheses involving exponential interarrival times (see Kim and Whitt (2014b) for a detailed comparison between the effectiveness of the two tests).

To perform CU KS test, for any interval $T_{i}=\left[a_{i}, b_{i}\right)$, let $t_{i j}, j=1, \ldots, k_{i}$, be the arrival times within the $i$-th interval obtained as the union over the $m$ weeks of the arrival times in each $T_{i}$. Now consider the rescaled arrival times defined by $\tau_{i j}=\frac{t_{i j}-a_{i}}{b_{i}-a_{i}}$. The rescaled arrival times, conditionally to the value $k_{i}$, are a collection of i.i.d. random variables uniformly distributed over $[0,1]$. Hence, in any interval, we compare the theoretical cumulative distribution function (cdf) $F(t)=t$ with the empirical cdf

$$
F_{i}(t)=\frac{1}{k_{i}} \sum_{j=1}^{k_{i}} \mathbf{1}_{\left\{\tau_{i j} \leq t\right\}}, \quad 0 \leq t \leq 1 .
$$

The test statistics is defined as follows

$$
D_{i}=\sup _{0 \leq t \leq 1}\left(\left|F_{i}(t)-t\right|\right) .
$$

The critical value for this test is denoted as $T\left(k_{i}, \alpha\right)$ and its values can be found on the KS test critical values table. Accordingly, the Poisson hypothesis is not rejected if

$$
D_{i} \leq T\left(k_{i}, \alpha\right), \quad i=1, \ldots, N .
$$

This test has to be satisfied on each interval $T_{i}$ to qualify the partition $P$ given by $\left\{T_{i}\right\}$ as feasible, in the sense that the CU KS test is satisfied, too.

A further restriction is imposed on the feasible partitions. Given the experimental data, realistic partitions can not have a granularity too fine to avoid that some $k_{i}$ being too small may unduly determine the rejection of the CU KS test. To this aim, a suited lower threshold value for the interval length must be chosen, taking into account the specific case study considered.

Now let us evaluate the feasible partitions also in terms of the characteristics of the function $\lambda_{D}(t)$. It would be amenable to define a fit error for $\lambda(t)$, which unfortunately is unknown. The problem can be resolved by considering a piecewise constant approximation $\lambda_{F}(t)$ over a very fine partition $P_{F}$ of $T$. A set of 96 equally spaced intervals of 15 minutes was considered and the corresponding average rates $\lambda_{i}^{F}$ were estimated from data.

The function $\lambda_{F}(t)$ can be considered as an empirical arrival rate model. Note that partition $P_{F}$ need not be feasible since it only serves to define the finest piecewise constant approximation of $\lambda(t)$. Therefore the following fit error can be defined

$$
E(P)=\sum_{i=1}^{N} \sum_{j=1}^{N_{j}}\left(\lambda_{j}-\lambda_{i_{j}}^{F}\right)^{2},
$$


where $N_{j}$ is the number of intervals of 15 minutes contained in $T_{j}$, and identified by the set of indexes $\left\{i_{j}\right\} \subset\{1, \ldots, 96\}$.

Finally, it is also advisable to characterize the "smoothness" of any approximation $\lambda_{D}(t)$ to avoid very gross partitions with high jumps between adjacent intervals using the mean squared error

$$
S(P)=\sum_{j=2}^{N}\left(\lambda_{j}-\lambda_{j-1}\right)^{2} .
$$

In the following Sect. 2.2 the model features illustrated above are organized in a proper optimization procedure that provides the selection of the best partition according to conflicting goals.

The approach we propose enables us to well address the major two issues raised in Kim and Whitt (2014a) (and reported in the Introduction) when dealing with modelling ED patient arrivals, namely the choice of the intervals and the overdispersion. Concerning the third issue, the data rounding, the arrival times in the data we collected are rounded to seconds (format $\mathrm{hh}: \mathrm{mm}: \mathrm{ss}$ ), and occurrences of simultaneous arrivals which would cause zero interarrival times are not present. Therefore, we do not need any unrounding procedure. Anyhow, as already pointed out above, the CU KS test we use is not very sensitive to data rounding.

\subsection{Statement of the optimization problem}

Any partition $P=\left\{T_{i}\right\}$ of $T=[0,24]$ is characterized by the boundary points $\left\{x_{i}\right\}$ of its intervals and by their number $N$. Let us introduce a vector of variables $x \in \mathbb{Z}^{25}$ such that

$$
T_{i}=\left[x_{i}, x_{i+1}\right),
$$

$i=1, \ldots, 24$, with $x_{1}=0$ and $x_{25}=24$.

Functions in (5) and (6) are indeed functions of $x$, and therefore will be denoted by $E(x)$ and $S(x)$, respectively. Therefore, the objective function that constitutes the selection criterion is given by

$$
f(x)=E(x)+w S(x),
$$

where $w>0$ is a parameter that controls the weight of the smoothness penalty term compared to the fit error: the larger $w$, the smaller the difference between average arrival rates in adjacent intervals; this, in turn, implies that on a steep section of $\lambda_{F}(t)$ an increased number of shorter intervals is adopted to fill the gap with relatively small jumps.

The set $\mathcal{P}$ of feasible partitions is defined as follows: 


$$
\begin{aligned}
& \mathcal{P}=\left\{x \in \mathbb{Z}^{25} \mid x_{1}=0, \quad x_{25}=24, \quad x_{i+1}-x_{i} \geq \ell_{i}, \quad g_{i}(x) \leq 0,\right. \\
& \left.h_{i}(x) \leq 0, \quad i=1, \ldots, N\right\}
\end{aligned}
$$

where

$$
\begin{gathered}
\ell_{i}= \begin{cases}0 & \text { if } x_{i}=x_{i+1}, \\
\ell & \text { otherwise, }\end{cases} \\
g_{i}(x)= \begin{cases}0 & \text { if } x_{i}=x_{i+1}, \\
D_{i}-T\left(k_{i}, \alpha\right) & \text { otherwise, }\end{cases} \\
h_{i}(x)=\left\{\begin{array}{lll}
0 & \text { if } x_{i}=x_{i+1}, \\
D s_{i}-\chi_{m-1, \alpha}^{2} & \text { otherwise },
\end{array}\right.
\end{gathered}
$$

$i=1, \ldots, N$. The value $\ell$ in (9) denotes the minimum interval length allowed and we assume $\ell \geq 1 / 4$. Of course, constraints $g_{i}(x) \leq 0$ represent the satisfaction of the CU KS test in (4), while constraints $h_{i}(x) \leq 0$ concern the dispersion test in (2). Therefore, the best piecewise constant approximation $\lambda_{D}^{\star}(t)$ of the time-varying arrival rate $\lambda(t)$ is obtained by solving the following black-box optimization problem:

$$
\begin{aligned}
& \max f(x) \\
& \text { s.t. } x \in \mathcal{P} .
\end{aligned}
$$

We highlight that the idea of using as constraints of the optimization problem a test to validate the underlying statistical hypothesis on data along with a dispersion test is completely novel in the framework of modeling the ED patient arrivals process. The only proposal which uses a similar approach is in our previous paper (De Santis et al. 2020).

It is important to note that in (7) the objective function has no analytical structure in terms of the independent variables and it can only be computed by a datadriven procedure once the $x_{i}$ 's values are given. The same is true for the constraints $g_{i}(x)$ and $h_{i}(x)$ in (8). Therefore the problem at hand is an integer nonlinear constrained black-box problem, and both the objective function and the constraints are relatively expensive to compute and this makes it difficult to efficiently solve. Consequently, classical optimization methods either can not be applied (since based on the analytic knowledge of the functions involved) or they are not efficient especially when evaluating the functions at a given point is very computationally expensive. Therefore to tackle the problem (12) we turned our attention to the class of Derivative-Free Optimization and black-box methods (see, e.g., Audet and Hare (2017), Conn et al. (2009), Larson et al. (2019)). Specifically, we adopt the algorithmic framework recently proposed in Liuzzi et al. (2020). It represents a novel strategy for solving black-box problems with integer variables and it is based on the use of suited search directions and a non-monotone line search procedure. Moreover, it 
Table 1 The total number of arrivals, for each weekday, over the 13 considered weeks

\begin{tabular}{lllllll}
\hline Mon. & Tues. & Wed. & Thur. & Fri. & Sat. & Sun. \\
\hline 2140 & 2114 & 2078 & 2040 & 2139 & 1995 & 1745 \\
\hline
\end{tabular}

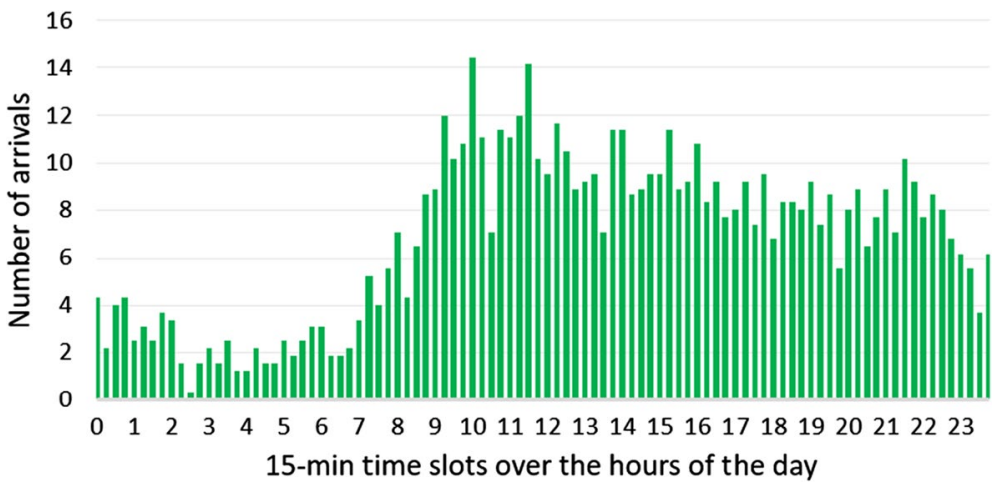

Fig. 1 Plot of the daily average arrival rate $\lambda_{i}^{F}, i=1, \ldots, 96$, over the 13 considered weeks

can handle generally-constrained problems by using a penalty approach. We refer to Liuzzi et al. (2020) for a detailed description and we only highlight that the results reported in Liuzzi et al. (2020) clearly show that this algorithm framework is particularly efficient in tackling black-box problems like the one in (12). In particular, the effectiveness of the adopted exploration strategy concerning the state-of-the-art methods for black-box is shown. This is because the approach proposed in Liuzzi et al. (2020) combines computational efficiency with a high level of reliability.

\section{The case study under consideration}

The case study we consider concerns the ED of Policlinico Umberto I, a very large hospital in Rome, Italy. It is the biggest ED in the Lazio region in terms of yearly patients arrivals (about 140,000 on average). Thanks to the cooperation of the ED staff, we were able to collect data concerning patient arrivals for the whole year 2018. In particular, for this work, we focus on the patients' arrival data collected in the first 13 weeks of the year, i.e. on data collected from the 1st of January to the 31st of March. Both walk-in patients and patients transported by emergency medical service vehicles are considered. The total number of arrivals, for each weekday, over the 13 weeks is reported in Table 1.

In Fig. 1 the plot of the average rates $\lambda_{i}^{F}$ estimated from data over 96 equally spaced intervals of 15 minutes is reported.

From this figure, it can be easily observed that, as expected, the arrival rate drastically changes from night hours to day hours, with significant growth during the morning hours. 


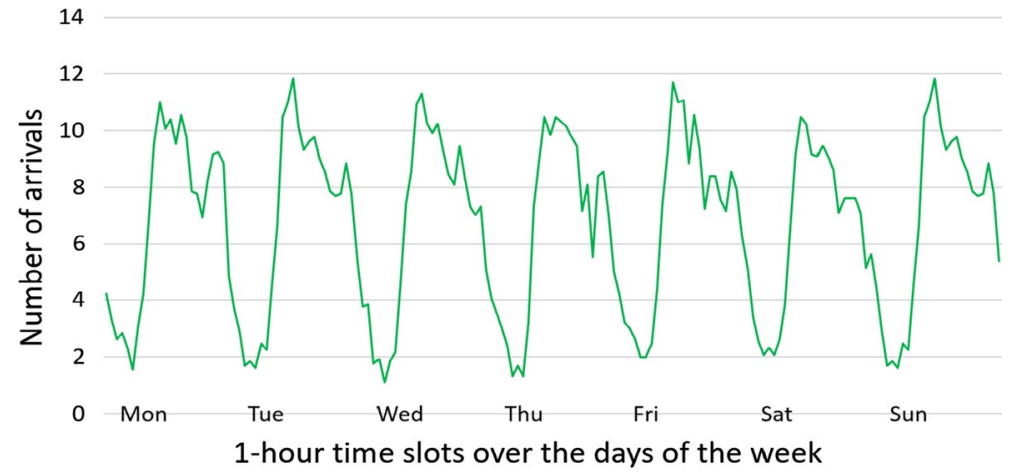

Fig. 2 Plot of the weekly average arrival rate for the 13 considered weeks

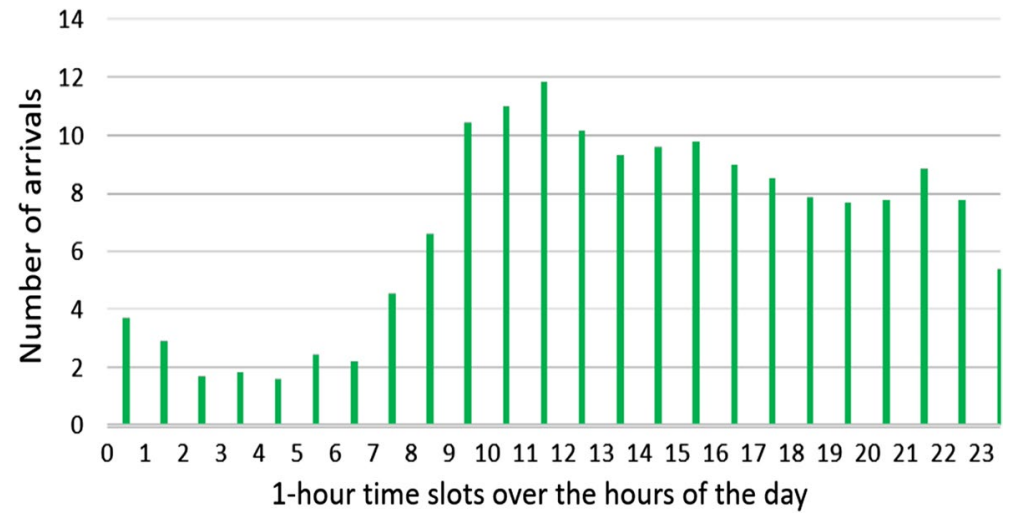

Fig. 3 Plot of the average hourly arrival rate for the Tuesdays over the 13 considered weeks of the year

In Fig. 2, the weekly hourly average arrival rate obtained by averaging the number of arrivals occurring in the same hourly time slot over the 13 considered weeks is reported.

It is worth highlighting that, following the literature (see, i.e., Kim and Whitt (2014a)), the average arrival rates among the days of the week are significantly different. Therefore, since averaging over these days would lead to inaccurate results, the different days of the week must be considered separately. Specifically, we can choose any day of the week to apply the methodology under study and the same way would apply to other days, thus obtaining a different partition for each day. As an example, we choose Tuesday. 


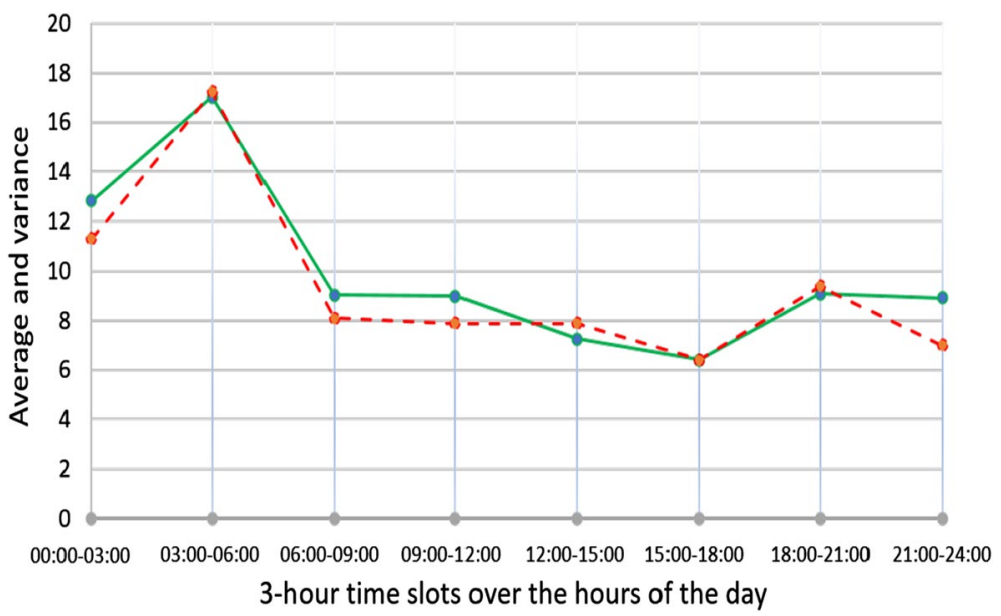

Fig. 4 Plot of the average (in solid green) and variance (in dashed red) of the interarrival times for the first Tuesday of year 2018. On the abscissa axis, 3-h time slots are considered

In Fig. 3, the plot of the hourly average arrival rate for the Tuesdays over the 13 considered weeks is reported, while Fig. 4 shows the mean and variance of the interarrival times that occurred on the first Tuesday of the year 2018 .

From this latter figure, we observe that these two statistics have similar values within each 3-h time slot and this complies with the property of the Poisson probability distribution for which mean and variance coincide.

We finally remark that seasonal phenomena might affect the number of weeks to be considered for model estimation due to large variability over successive weeks. Indeed, the overdispersion phenomenon may require a model calibration for each particular period of the year to take into account typical situations which occur, for instance, during flu season. This important aspect clearly emerges also from our experimentation reported in the next section.

\section{Experimental results}

In this section, we report the results of extensive experimentation on data concerning the case study described in Sect. 3, namely the ED patient arrivals collected in the first $m$ weeks of the year 2018. Different values of the number $m$ of weeks have been considered. Standard significance level $\alpha=0.05$ is used in the CU KS and dispersion tests.

In the optimization problem at hand the value of $\ell$ in (9) is set to 1 hour. Moreover, it is important to note that different values of the weight $w$ in the objective function (7) lead to various piecewise constant approximations with different fitting accuracy and degree of regularity. Therefore, we performed a careful tuning of this parameter, aiming at determining a value that represents a good trade-off between a small fit error and the smoothness of the approximation. 
In our experimentation, we used the default parameter values of the optimization algorithm adopted in Liuzzi et al. (2020). The stopping criterion is based on the maximum number of function evaluations set to 5000. As starting point $x^{0}$ of the optimization algorithm we adopt the following

$$
x_{i}^{0}=i-1, \quad i=1, \ldots, 25,
$$

which corresponds to the case of 24 intervals of unitary length. This choice is a commonly used partition in most of the approaches proposed in the literature (see e.g., Ahalt et al. (2018), Kim and Whitt (2014a)).

We used R language and all the runs were performed on a PC with an Intel Core i7-2600 quad-core 3.4 GHz Processor and 16 GB RAM.

Table 3 in the "Appendix" reports the results of CU KS and dispersion tests applied to the partition corresponding to the starting point $x^{0}$, considering $m=13$ weeks. In particular, in Table 3 for each one-hour slot the sample size $k_{i}$ is reported along with the $p$-value and the rejection/not rejection of the null hypothesis of the corresponding test. We observe that the arrivals are not overdispersed in any interval of the partition corresponding to $x^{0}$, i.e. all the constraints $h_{i}(x) \leq 0$ are satisfied and this allows us to combine data for the same day of the week over successive weeks. However, this partition is even infeasible, i.e., $g_{i}(x)>0$, for some $i$; this corresponds to reject the statistical hypothesis on some $T_{i}$. Notwithstanding, even if the starting point is infeasible, the optimization algorithm we use can find an optimal solution.

As we already mentioned, the choice of a proper value for the weight $w$ in the objective function (7) is important and not straightforward. On the other hand, the number $m$ of weeks considered also affects both the accuracy of the approximation, through the average rates estimated on each interval, and the consistency of the results, which is ensured by constraints (10) and (11). However, while $w$ is related to the statement of the optimization problem (12) and it can be arbitrarily chosen, the choice of $m$ is strictly connected to the available data. In (Kim and Whitt 2014a, Section 4), the authors assert that having 10 arrivals in the one-hour slot 9-10 a.m., it is necessary to combine data over 20 weeks in order to have a sufficient sample size (200 patient arrivals). However, being their approach based on equally spaced intervals, one-hour slots are also adopted during off-peak hours, for instance during the night. This implies that the sample size corresponding to data combination over 20 weeks for these slots could no longer be sufficient to guarantee good results. This is clearly pointed out in Table 3 in the "Appendix" where the sample size $k_{i}$ corresponding to some of the one-hour night slots is very low considering $m=13$ weeks and it remains insufficient even if 26 weeks are considered (see subsequent Table 5). The approach we propose overcomes this drawback since, for each choice of $m$, we determine the length of the intervals by solving the optimization problem (12). Of course, there could be values of $m$ such that problem (12) does not have feasible solutions, i.e. a partition such that the NHPP hypothesis holds and the results are consistent does not exists for such $m$.

To give an idea of the computational burden required by the application of our approach, we report the CPU elapsed time corresponding to one function/constraints 
Table 2 CPU elapsed time corresponding to one function/constraints evaluation for different values of $m$

\begin{tabular}{lllllll}
\hline$m$ & 4 & 9 & 13 & 17 & 22 & 26 \\
\hline Time (s) & 13.01 & 16.12 & 26.07 & 28.10 & 36.21 & 44.14 \\
\hline
\end{tabular}

evaluation used for solving the optimization problem 12. Table 2 indicates this time (in seconds) for different values of the parameter $m$.

This table evidences the increase of the computational effort required as the parameter $m$ increases. We believe that the choice $m=13$ represents a good trade-off between the accuracy of the results and computing time.

Now, to deeper examine how the parameters $w$ and $m$ affect the optimal partition, we performed a sensitivity analysis, focusing first on the case with fixed $m$ and $w$ varying. In particular, we have chosen to focus on $m=13$ weeks since, as discussed above, in this case, an overly computational burden is not required and anyhow we expect that no substantial changes in the conclusions would be obtained with different values of $m$. This is confirmed by further experimentation whose results are not reported here for the sake of brevity.

This analysis allows us to obtain several partitions that may be considered for a proper fine-tuning of $w$. In particular, we consider different values of $w$ within the set $\left\{0,0.1,1,10,10^{3}\right\}$. Table 4 in the "Appendix" reports the optimal partitions obtained by solving the problem (12) for these values of $w$. In particular, Table 4 includes the intervals of the partition, the value of the sample size $k_{i}$ corresponding to each interval over 13 weeks and the results of the CU KS and dispersion tests, namely the $p$-value and the rejection/not rejection of the null hypothesis of the corresponding test.

In Fig. 5, for graphical comparison, we report the plots of the empirical arrival rate model $\lambda_{F}(t)$ and its piecewise constant approximation $\lambda_{D}(t)$ corresponding to the optimal partitions obtained.

Two effects can be clearly observed as $w$ increases: on the one hand, on steep sections of $\lambda_{F}(t)$, shorter intervals are adopted to reduce large gaps between adjacent intervals; on the other hand, when $\lambda_{F}(t)$ is approximately flat, a lower number of intervals may be sufficient to guarantee small gaps. This is confirmed by the two top plots in Fig. 5 which correspond to $w=0$ and $w=0.1$. In fact, in the first plot $(w=0)$, where only the fit error is included in the objective function, and in the second one $(w=0.1)$, where anyhow the fit error is the dominant term of the objective function, the optimal partition is composed of a relatively large number of intervals. In particular, in the partition corresponding to $w=0.1$, fewer intervals are adopted during the daytime. As expected, a smaller number of intervals is attained when $w=1, w=10$ and $w=10^{3}$. Note that, since on the steep section corresponding to the time slot 7:00-10:00 a.m. the maximum number of allowed intervals (due to the lower threshold value of one hour given by the choice $\ell=1$ in (9)) is already used, the only way to decrease the smoothness term of the objective function is to enlarge the intervals during both the day and the night. It is worth noting that for $w=10^{3}$, the number of intervals increases if 
Fig. 5 Graphical comparison between the empirical arrival rate model $\lambda_{F}(t)$ (in green) and the piecewise constant approximation $\lambda_{D}(t)$ (in red) corresponding to the optimal partition obtained by solving the problem (12) (with $m=13$ ) for different values of the parameter $w$. From top to bottom: $w=0,0.1,1,10,10^{3}$ (Color figure online)
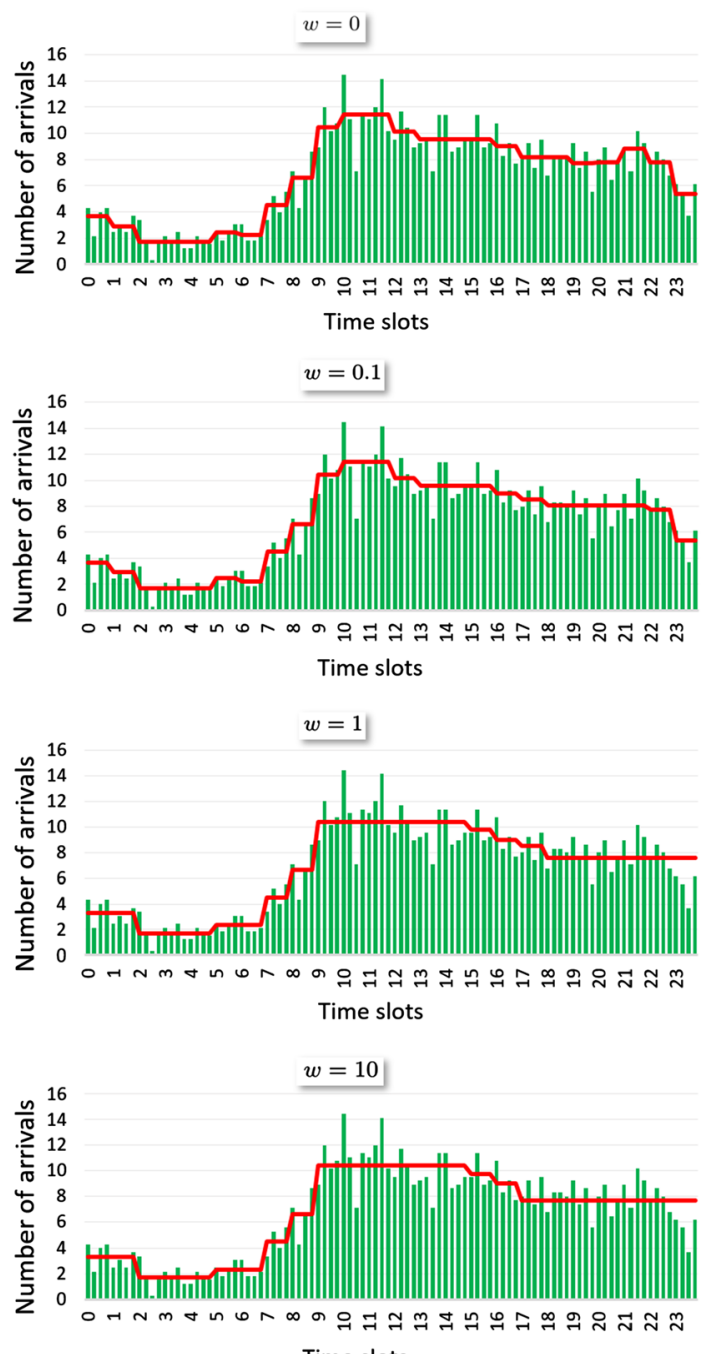

Time slots

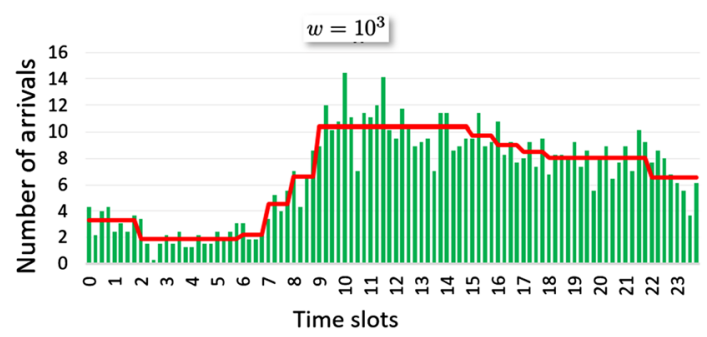


compared with the case $w=10$. This occurs to offset the increase in the fit error term due to the use of a smaller number of intervals on the flatter sections. As a consequence, the partition has an unexpected interval at the end of the day.

We point out that for each value of $w$, the optimization algorithm finds an optimal partition (of course feasible concerning all the constraints), despite some constraints related to the CU KS test are violated at the initial partition, i.e. the one corresponding to $x^{0}$ in (13), namely the standard assumption of one-hour slots usually adopted. This means that the data used, comply with the NHPP hypothesis and are sufficient to appropriately define the piecewise constant approximation of the ED arrival rate.

Conversely, when the optimization algorithm does not find a feasible partition, the CU KS test or the dispersion test related to some $T_{i}$ are never satisfied. This implies that the process is not conforming to the NHPP hypothesis or that the data are overdispersed. This is clearly highlighted by our subsequent experimentation where we set $w=1$, letting $m$ varying within the set $\{5,9,17,22,26\}$.

First, in Table 5 in the "Appendix" we report the results of CU KS and dispersion tests applied to the partition corresponding to the starting point $x^{0}$ in (13), for these different values of $m$. Once more, this table evidences that the use of equally spaced intervals of one-hour length during the whole day can be inappropriate. As an example, see the results of the tests on the time slot 02:00-03:00. Moreover, note that, for all these values of $m$, the initial partition corresponding to the starting point $x_{0}$ is infeasible, except when $m=5$. Indeed, the constraints corresponding to CU KS and dispersion tests are violated for some $T_{i}$, meaning that the validity of the standard assumption of one-hour time slots strongly depends on the time period considered for using the collected data. To this aim, a strength of our approach is its ability to assist in the selection of a reasonable value for $m$. If there is no value of $m$ such that the optimization algorithm determines an optimal solution (due to infeasibility), then it may be inappropriate to consider the ED arrival process in hand as NHPP.

The subsequent Table 6 in the "Appendix" includes the optimal partitions obtained by solving problem the (12) for the considered values of $m \in\{5,9,17,22,26\}$. Like the previous tables, Table 6 includes the intervals of the partition, the value of the sample size $k_{i}$ corresponding to each interval and the results of CU KS and dispersion tests. For all the considered values of $m$, the optimization algorithm determines an optimal solution with the only exception of $m=26$. In this latter case, the maximum number of function evaluations allowed is not enough to compute an optimal solution: in fact, we obtain an infeasible solution since the CU KS test related to the last interval of the day is not satisfied. This could be partially unexpected since more accurate results should be obtained when considering greater sample size. However, by adding the last four weeks (passing from $m=22$ to $m=26$ ) which corresponds to June, the data become affected by a seasonal trend and the NHPP assumption is no longer valid. This confirms the remark reported at the end of Sect. 3 about the need of model calibration for possibly taking into account seasonality.

In Fig. 6 we report a graphical comparison between the empirical arrival rate model $\lambda_{F}(t)$ and the piecewise constant approximation $\lambda_{D}(t)$ corresponding to the optimal partitions obtained for the considered values of $m$. We observe that the variability of $\lambda_{F}(t)$ reduces as the value of $m$ increases since averaging on more 
Fig. 6 Graphical comparison between the empirical arrival rate model $\lambda_{F}(t)$ (in green) and the piecewise constant approximation $\lambda_{D}(t)$ (in red) corresponding to the optimal partition obtained by solving the problem (12) (with $w=1$ ) for different values of the parameter $m$. From top to bottom: $m=5,9,17,22,26$ (Color figure online)
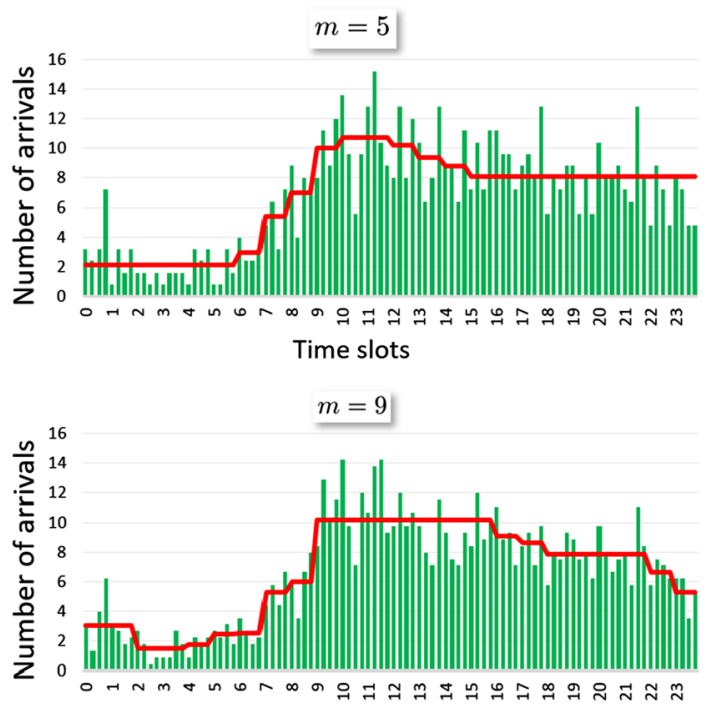

Time slots

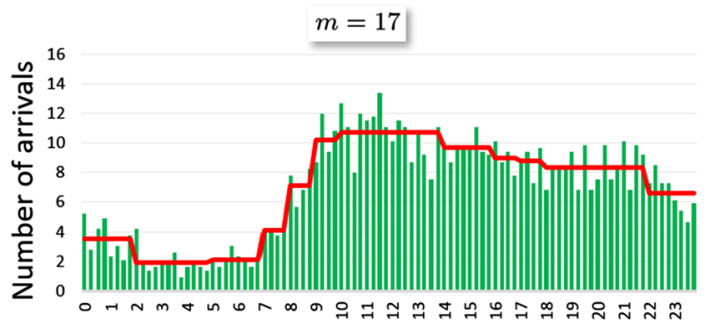

Time slots

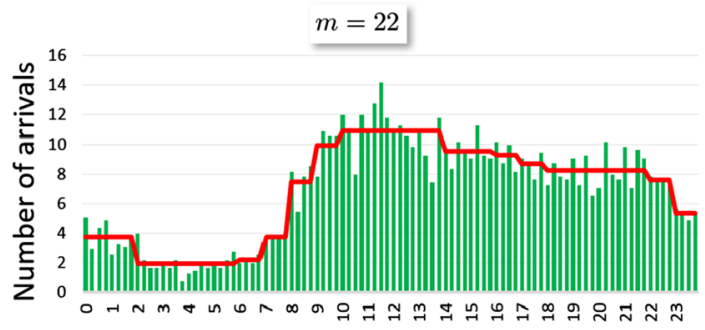

Time slots

data leads to flattening the fluctuation. Despite these rapid oscillations and unlike the other considered values of $m$, for $m=5$ the empirical model $\lambda_{F}(t)$ shows a constant trend during both the night and day hours. This results in a piecewise constant approximation $\lambda_{D}(t)$ that is flat in all the time slots of the $24 \mathrm{~h}$ of the day except the ones related to the morning hours, for which many intervals are used. To guarantee a good fitting error between $\lambda_{D}(t)$ and $\lambda_{F}(t)$, it would be necessary to use shorter intervals, but this is not allowed by the choice $\ell=1$ in the 
constraints (9). For the other considered values of $m$, the number of intervals increases, leading to partitions that improve the fitting error if compared with the case $m=5$. In particular, we observe that the piecewise constant approximation $\lambda_{D}(t)$ obtained for $m=22$ benefits from the lower fluctuations resulting from averaging more data. Therefore, as expected, using the maximum number of available data leads to the most accurate piecewise constant approximation. However, when considering too much data, seasonal phenomena could give rise to the rejection of the null hypothesis of the considered tests, as observed for the case $m=26$. Moreover, as highlighted at the end of Section 5 in Kim and Whitt (2014a), a tendency to reject the NHPP hypothesis (i.e. the null hypothesis of the CU KS test) may be encountered when the sample size is large. In fact, a larger sample size requires stronger evidence of the null hypothesis for the test to be passed. Notwithstanding, our approach can overcome these drawbacks, providing us with an optimal strategy to identify the best way of using the collected data.

\section{A preliminary assessment}

As we already mentioned, this paper represents the first step toward the development of a complete and accurate DES model of the ED under study, thus it is only focused on modeling patient arrivals. However, it could be interesting to preliminary assess the impact of the method we propose in modeling the overall patient flow through the ED. Unfortunately, at this stage, we do not have detailed information concerning all the many processes inside the ED after triage, hence we are still unable to construct a complete simulation model of the ED. On the other hand, for evaluating the actual consequences of the proposed arrival process approximation, it is enough to construct a simplified model which includes only patient arrivals and triage, being the latter the first process encountered by a patient after arrival and the one most affected by patient arrivals process. This, of course, must be considered only a preliminary assessment.

In this simplified model, triage is modeled as "seize-delay-release" process where the seized resource is a dedicated nurse and the delay (the triage time in hours) is assumed distributed according to the Weibull distribution $\operatorname{WEIBULL}(\alpha, \beta)$ with $\alpha=3$ and $\beta=0.1$. Such distribution and its parameters have been obtained using a statistical analysis of available data on triage process times. The model has been implemented by using Ucar et al. (2019), a process-oriented and trajectory-based DES package for $\mathrm{R}$ language. In running simulations, we used the following setting: 30 independent replications each of 14 weeks length and warming-up period of 1 week. 


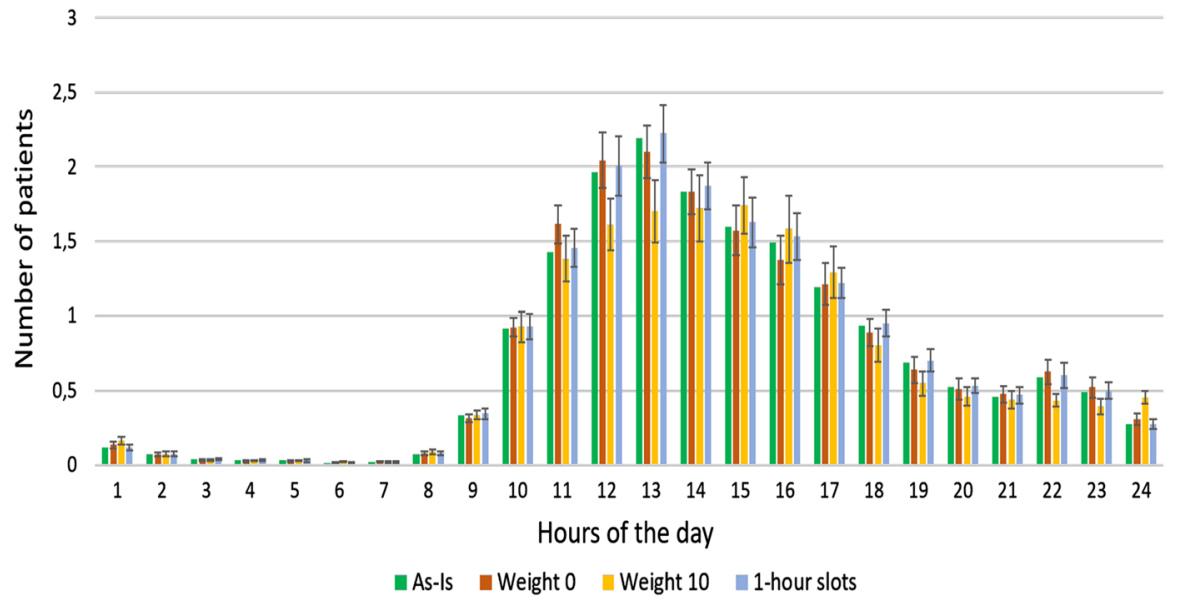

Fig. 7 Average number of patient queued waiting for the triage

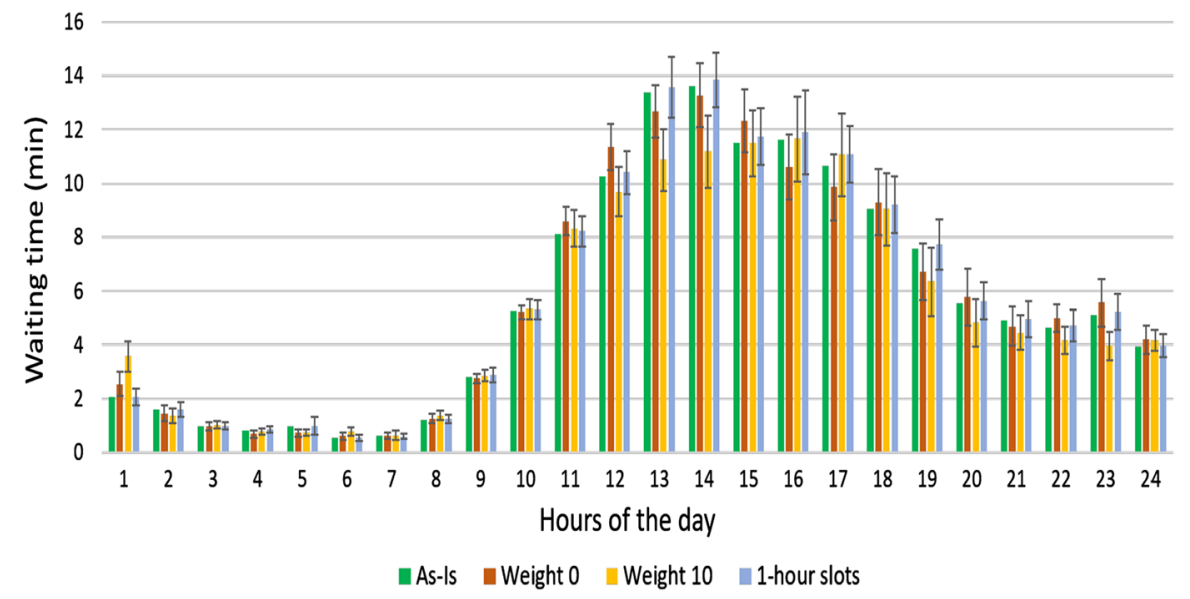

Fig. 8 Average of waiting times before the triage

In order to perform a significant assessment of the method we propose for patient arrivals, we compared results obtained by the following scenarios:

- use of real data for generating patients arrivals for the first 13 weeks of the year 2018; 
- use of our method (with different choices of the weight $w$ ) for defining the NHPP process of patient arrivals;

- use of the standard method which considers 24 one-hour intervals for defining the NHPP process of patient arrivals.

We choose to use as KPIs in this comparison those mostly affected by the arrival process, namely

- average number of patients queued waiting for the triage;

- average patient waiting time before the triage.

In Figs. 7 and in 8 we report these KPIs on an hourly basis. In particular, we report results obtained by the real data (as-is status), those obtained using our approach (using $w=0$ and $w=10$ ) and the ones derived from the partition with 24 one-hour intervals.

We can observe from both figures, that the finer discretizations, i.e. those corresponding to one-hour slots and to $w=0$ (18 intervals), provide results closer to the real process. On the other hand, the grosser partition obtained with $w=10$, corresponding to 10 intervals, maintains a good fit with the real process, noticing that a small decrease in accuracy is evidenced in time interval 11:00-13:00 where the real data feature high variability while the partition provided by our method with $(w=10)$ generates a wide interval with a constant rate from 9:00 to 14:00 (see Fig. 5). Therefore a good accuracy is obtained with a limited number of intervals that provide the management with a better scenario for planning the resource allocation for the ED services.

\section{Conclusions}

In this work, we examined the arrival process to EDs by providing a novel methodology that can improve the reliability of the modeling approaches frequently used to deal with this complex system, i.e. the Discrete Event Simulation modeling. Following the literature, we adopted the standard assumption of representing the ED arrival process as an NHPP, which is suitable for modeling strongly time-varying processes. In particular, the final goal of the proposed approach is to accurately estimate the unknown arrival rate, i.e. the time-dependent parameter of the NHPP, by using a reasonable piecewise constant approximation. To this aim, an integer nonlinear black-box optimization problem is solved to determine 
the optimal partition of the $24 \mathrm{~h}$ into a suitable number of non equally spaced intervals. To guarantee the reliability of this estimation procedure, two types of statistical tests are considered as constraints for each interval of any candidate partition: the CU KS test must be satisfied to ensure the consistency between the NHPP hypothesis and the ED arrivals; the dispersion test must be satisfied to avoid the overdispersion of data. To the best of our knowledge, our methodology represents the first optimization-based approach adopted for determining the best stochastic model for the ED arrival process.

The extensive experimentation we performed on data collected from an ED of a big hospital in Italy, shows that our approach can find a piecewise constant approximation which represents a good trade-off between a small fit error with the empirical arrival rate model and the smoothness of the approximation. This result is accomplished by the optimization algorithm, despite some constraints at the starting point, which corresponds to the commonly adopted partition composed of one-hour time slots, are violated. Moreover, some significant sensitivity analyses are performed to investigate the fine-tuning of the two parameters affecting the quality of the piecewise constant approximation: the weight of the smoothness of the approximation in the objective function (concerning the fit error) and the number of weeks considered from the arrivals data. While the former can be arbitrarily chosen by a user according to the desired level of smoothness, the latter affects the accuracy of the arrival rate estimation. In general, the more weeks are considered, the more accurate is the arrival rate approximation, as long as the NHPP assumption still holds and the data do not become overdispersed.

Further experimentation allowed us to perform a preliminary assessment of the proposed approach, monitoring the number of patients queued at the triage and the corresponding waiting time obtained in a simplified simulation model representing only the initial passenger flow through the ED, namely the arrivals and the triage processes. The results showed that it is possible to adopt a grosser partition of the $24 \mathrm{~h}$, which is preferable to the management point of view, still ensuring a good fit with the real data process when compared with that of a plain partition with shorter equally spaced intervals.

As regards future work, to deeper analyze the robustness of the proposed approach, we could use alternative statistical tests, such as the Lewis and the Log tests described in Kim and Whitt (2014a), in place of the CU KS test. Moreover, whenever Discrete Event Simulation modeling is the chosen methodology to study ED operation, a model calibration approach could be also used to determine the best value of the weight used in the objective function to penalize the "smoothness term". The optimal value of this parameter could be obtained by minimizing the deviation 
between the simulation outputs and the corresponding key performance indicators computed through the data. This enables to obtain a representation of the ED arrival process that leads to an improved simulation model of the system under study.

\section{Appendix}

In this "Appendix" we report the detailed results of the CU KS and dispersion tests related to the partitions considered throughout the paper (Tables 3, 4, 5, 6).

Table 3 Results of the CU $\mathrm{KS}$ and dispersion tests (with a significance level of 0.05 ) applied to each interval of the partition corresponding to the starting point $x^{0}$

\begin{tabular}{|c|c|c|c|c|c|}
\hline \multirow[t]{2}{*}{ Interval } & \multirow[t]{2}{*}{$k_{i}$} & \multicolumn{2}{|c|}{ CU KS test } & \multicolumn{2}{|c|}{ Dispersion test } \\
\hline & & $p$-value & $H_{0}$ & $p$-value & $H_{0}$ \\
\hline 0:00 - 01:00 & 48 & 0.836 & Not $\mathrm{r}$ & 0.801 & Not \\
\hline $1: 00-02: 00$ & 38 & 0.950 & ed & ( & ed \\
\hline $2: 00-03: 00$ & 22 & 0.027 & $\mathrm{R}$ & & ted \\
\hline $3: 00-04: 00$ & 24 & 0.752 & & & ed \\
\hline $4: 00-05: 00$ & 21 & 0 & ed & 0 & ted \\
\hline $5: 00-06: 00$ & 32 & 0.312 & $d$ & + & ed \\
\hline $6: 00-07: 00$ & 29 & 0 & d & 0.538 & ted \\
\hline 07:00 - 08:00 & 59 & 0 & $\mathrm{~N}$ & & ted \\
\hline 08:00 - 09:00 & 86 & 0.393 & d & 0.734 & ed \\
\hline 09:00 - 10:00 & 136 & & & & ted \\
\hline $10: 00-$ & 143 & 0 & $\mathrm{~F}$ & 0.966 & ed \\
\hline $11: 00-12: 00$ & 154 & 0 & & 0 & cted \\
\hline $12: 00-13: 00$ & 132 & 0 & $\mathrm{~N}$ & 0 & cted \\
\hline $13: 00-$ & 121 & 0 & N & 0.984 & cted \\
\hline $14: 00-15: 00$ & 125 & 0.885 & Not & 0.500 & Not rejected \\
\hline $15: 00-16: 00$ & 127 & 0.928 & d & 0.610 & cted \\
\hline $16: 00-17: 00$ & 117 & 0.479 & Not rejected & 0.987 & Not rejected \\
\hline $17: 0$ & 111 & 0 & r & & ed \\
\hline $18: 00-19: 00$ & 102 & 0.458 & Not rejected & 0.912 & Not rejected \\
\hline $19: 00-20: 00$ & 100 & 0 & $\mathrm{~d}$ & 21 & ed \\
\hline $20: 00-21: 00$ & 101 & 0 & $\mathrm{~N}$ & 0 & Not rejected \\
\hline $21: 00-22: 00$ & 115 & 0.763 & Not & 0.604 & Not \\
\hline 00 & 101 & 0 & $\mathrm{~N}$ & 0 . & ed \\
\hline $23: 00-24: 00$ & 70 & 0.864 & Not rejected & 0.104 & Not rejected \\
\hline
\end{tabular}

The considered number of weeks is $m=13$. For each interval of each partition, the sample size of the dispersion test is $m . H_{0}$ denotes the null hypothesis of the corresponding test 
Table 4 Results of the CU KS and dispersion tests (with a significance level of 0.05) applied to each interval of the optimal partition obtained by solving problem (12) for different values of the parameter $w$, with $m$ fixed to 13 weeks

\begin{tabular}{|c|c|c|c|c|c|c|}
\hline \multirow[t]{2}{*}{$w$} & \multirow[t]{2}{*}{ Interval } & \multirow[t]{2}{*}{$k_{i}$} & \multicolumn{2}{|c|}{ CU KS test } & \multicolumn{2}{|c|}{ Dispersion test } \\
\hline & & & $p$-value & $H_{0}$ & $p$-value & $H_{0}$ \\
\hline \multirow[t]{18}{*}{0} & 00:00 - 01:00 & 48 & 0.836 & Not rejected & 0.801 & Not rejected \\
\hline & $01: 00-02: 00$ & 38 & 0.950 & Not rejected & 0.450 & Not rejected \\
\hline & $02: 00-05: 00$ & 67 & 0.504 & Not rejected & 0.100 & Not rejected \\
\hline & 05:00 - 06:00 & 32 & 0.312 & Not rejected & 0.524 & Not rejected \\
\hline & $06: 00-07: 00$ & 29 & 0.634 & Not rejected & 0.538 & Not rejected \\
\hline & 07:00 - 08:00 & 59 & 0.424 & Not rejected & 0.252 & Not rejected \\
\hline & 08:00 - 09:00 & 86 & 0.393 & Not rejected & 0.734 & Not rejected \\
\hline & $09: 00-10: 00$ & 136 & 0.635 & Not rejected & 0.803 & Not rejected \\
\hline & $10: 00-12: 00$ & 297 & 0.433 & Not rejected & 0.994 & Not rejected \\
\hline & $12: 00-13: 00$ & 132 & 0.858 & Not rejected & 0.948 & Not rejected \\
\hline & $13: 00-16: 00$ & 373 & 0.958 & Not rejected & 0.502 & Not rejected \\
\hline & $16: 00-17: 00$ & 117 & 0.479 & Not rejected & 0.987 & Not rejected \\
\hline & $17: 00-19: 00$ & 213 & 0.999 & Not rejected & 0.937 & Not rejected \\
\hline & $19: 00-20: 00$ & 100 & 0.095 & Not rejected & 0.527 & Not rejected \\
\hline & $20: 00-21: 00$ & 101 & 0.656 & Not rejected & 0.586 & Not rejected \\
\hline & $21: 00-22: 00$ & 115 & 0.763 & Not rejected & 0.604 & Not rejected \\
\hline & $22: 00-23: 00$ & 101 & 0.916 & Not rejected & 0.305 & Not rejected \\
\hline & $23: 00-24: 00$ & 70 & 0.864 & Not rejected & 0.104 & Not rejected \\
\hline \multirow[t]{16}{*}{0.1} & $00: 00-01: 00$ & 48 & 0.836 & Not rejected & 0.801 & Not rejected \\
\hline & $01: 00-02: 00$ & 38 & 0.950 & Not rejected & 0.450 & Not rejected \\
\hline & $02: 00-05: 00$ & 67 & 0.504 & Not rejected & 0.100 & Not rejected \\
\hline & 05:00 - 06:00 & 32 & 0.312 & Not rejected & 0.524 & Not rejected \\
\hline & $06: 00-07: 00$ & 29 & 0.634 & Not rejected & 0.538 & Not rejected \\
\hline & 07:00 - 08:00 & 59 & 0.424 & Not rejected & 0.252 & Not rejected \\
\hline & 08:00 - 09:00 & 86 & 0.393 & Not rejected & 0.734 & Not rejected \\
\hline & $09: 00-10: 00$ & 136 & 0.635 & Not rejected & 0.803 & Not rejected \\
\hline & $10: 00-12: 00$ & 297 & 0.433 & Not rejected & 0.994 & Not rejected \\
\hline & $12: 00-13: 00$ & 132 & 0.858 & Not rejected & 0.948 & Not rejected \\
\hline & $13: 00-16: 00$ & 373 & 0.958 & Not rejected & 0.502 & Not rejected \\
\hline & $16: 00-17: 00$ & 117 & 0.479 & Not rejected & 0.987 & Not rejected \\
\hline & $17: 00-18: 00$ & 111 & 0.769 & Not rejected & 0.516 & Not rejected \\
\hline & $18: 00-22: 00$ & 418 & 0.660 & Not rejected & 0.987 & Not rejected \\
\hline & $22: 00-23: 00$ & 101 & 0.916 & Not rejected & 0.305 & Not rejected \\
\hline & $23: 00-24: 00$ & 70 & 0.864 & Not rejected & 0.104 & Not rejected \\
\hline
\end{tabular}


Table 4 (continued)

\begin{tabular}{|c|c|c|c|c|c|c|}
\hline \multirow[t]{2}{*}{$w$} & \multirow[t]{2}{*}{ Interval } & \multirow[t]{2}{*}{$k_{i}$} & \multicolumn{2}{|c|}{ CU KS test } & \multicolumn{2}{|c|}{ Dispersion test } \\
\hline & & & $p$-value & $H_{0}$ & $p$-value & $H_{0}$ \\
\hline \multirow[t]{10}{*}{1} & $00: 00-02: 00$ & 86 & 0.825 & Not rejected & 0.709 & Not rejected \\
\hline & $02: 00-05: 00$ & 67 & 0.504 & Not rejected & 0.100 & Not rejected \\
\hline & $05: 00-07: 00$ & 61 & 0.739 & Not rejected & 0.313 & Not rejected \\
\hline & $07: 00-08: 00$ & 59 & 0.424 & Not rejected & 0.252 & Not rejected \\
\hline & $08: 00-09: 00$ & 86 & 0.393 & Not rejected & 0.734 & Not rejected \\
\hline & $09: 00-15: 00$ & 811 & 0.073 & Not rejected & 0.955 & Not rejected \\
\hline & $15: 00-16: 00$ & 127 & 0.928 & Not rejected & 0.610 & Not rejected \\
\hline & $16: 00-17: 00$ & 117 & 0.479 & Not rejected & 0.987 & Not rejected \\
\hline & $17: 00-18: 00$ & 111 & 0.769 & Not rejected & 0.516 & Not rejected \\
\hline & $18: 00-24: 00$ & 589 & 0.059 & Not rejected & 0.922 & Not rejected \\
\hline \multirow[t]{9}{*}{10} & $00: 00-02: 00$ & 86 & 0.825 & Not rejected & 0.709 & Not rejected \\
\hline & $02: 00-05: 00$ & 67 & 0.504 & Not rejected & 0.100 & Not rejected \\
\hline & $05: 00-07: 00$ & 61 & 0.739 & Not rejected & 0.313 & Not rejected \\
\hline & $07: 00-08: 00$ & 59 & 0.424 & Not rejected & 0.252 & Not rejected \\
\hline & 08:00 - 09:00 & 86 & 0.393 & Not rejected & 0.734 & Not rejected \\
\hline & $09: 00-15: 00$ & 811 & 0.073 & Not rejected & 0.955 & Not rejected \\
\hline & $15: 00-16: 00$ & 127 & 0.928 & Not rejected & 0.610 & Not rejected \\
\hline & $16: 00-17: 00$ & 117 & 0.479 & Not rejected & 0.987 & Not rejected \\
\hline & $17: 00-24: 00$ & 700 & 0.063 & Not rejected & 0.720 & Not rejected \\
\hline \multirow[t]{11}{*}{$10^{3}$} & $00: 00-02: 00$ & 86 & 0.825 & Not rejected & 0.709 & Not rejected \\
\hline & $02: 00-06: 00$ & 99 & 0.451 & Not rejected & 0.162 & Not rejected \\
\hline & $06: 00-07: 00$ & 29 & 0.634 & Not rejected & 0.538 & Not rejected \\
\hline & $07: 00-08: 00$ & 59 & 0.424 & Not rejected & 0.252 & Not rejected \\
\hline & 08:00 - 09:00 & 86 & 0.393 & Not rejected & 0.734 & Not rejected \\
\hline & $09: 00-15: 00$ & 811 & 0.073 & Not rejected & 0.955 & Not rejected \\
\hline & $15: 00-16: 00$ & 127 & 0.928 & Not rejected & 0.610 & Not rejected \\
\hline & $16: 00-17: 00$ & 117 & 0.479 & Not rejected & 0.987 & Not rejected \\
\hline & $17: 00-18: 00$ & 111 & 0.769 & Not rejected & 0.516 & Not rejected \\
\hline & $18: 00-22: 00$ & 418 & 0.660 & Not rejected & 0.987 & Not rejected \\
\hline & $22: 00-24: 00$ & 171 & 0.053 & Not rejected & 0.681 & Not rejected \\
\hline
\end{tabular}

From top to bottom: $w=0,0.1,1,10,10^{3}$. For each interval of each partition, the sample size of the dispersion test is equal to $m . H_{0}$ denotes the null hypothesis of the corresponding test 
Table 5 Results of the CU KS and dispersion tests (with a significance level of 0.05) applied to each interval of the partition corresponding to the starting point $x^{0}$

\begin{tabular}{|c|c|c|c|c|c|c|}
\hline \multirow[t]{2}{*}{$m$} & \multirow[t]{2}{*}{ Interval } & \multirow[t]{2}{*}{$k_{i}$} & \multicolumn{2}{|c|}{ CU KS test } & \multicolumn{2}{|c|}{ Dispersion test } \\
\hline & & & $p$-value & $H_{0}$ & $p$-value & $H_{0}$ \\
\hline & 00:00 - 01:00 & 20 & 0.167 & Not rejected & 0.240 & Not rejected \\
\hline & $01: 00-02: 00$ & 11 & 0.616 & Not rejected & 0.151 & Not rejected \\
\hline & 02:00 - 03:00 & 7 & 0.887 & Not rejected & 0.160 & Not rejected \\
\hline & 03:00-04:00 & 7 & 0.892 & Not rejected & 0.683 & Not rejected \\
\hline & $04: 00-05: 00$ & 12 & 0.217 & Not rejected & 0.856 & Not rejected \\
\hline & $05: 00-06: 00$ & 8 & 0.426 & Not rejected & 0.219 & Not rejected \\
\hline & $06: 00-07: 00$ & 15 & 0.884 & Not rejected & 0.504 & Not rejected \\
\hline & 07:00 - 08:00 & 27 & 0.820 & Not rejected & 0.164 & Not rejected \\
\hline & 08:00 - 09:00 & 35 & 0.875 & Not rejected & 0.534 & Not rejected \\
\hline & 09:00 - 10:00 & 50 & 0.378 & Not rejected & 0.844 & Not rejected \\
\hline & $10: 00-11: 00$ & 48 & 0.083 & Not rejected & 0.884 & Not rejected \\
\hline & $11: 00-12: 00$ & 59 & 0.484 & Not rejected & 0.966 & Not rejected \\
\hline & $12: 00-13: 00$ & 51 & 0.594 & Not rejected & 0.765 & Not rejected \\
\hline & $13: 00-14: 00$ & 47 & 0.651 & Not rejected & 0.689 & Not rejected \\
\hline & $14: 00-15: 00$ & 44 & 0.817 & Not rejected & 0.412 & Not rejected \\
\hline & $15: 00-16: 00$ & 45 & 0.811 & Not rejected & 0.168 & Not rejected \\
\hline & $16: 00-17: 00$ & 47 & 0.679 & Not rejected & 0.987 & Not rejected \\
\hline & $17: 00-18: 00$ & 49 & 0.486 & Not rejected & 0.534 & Not rejected \\
\hline & $18: 00-19: 00$ & 37 & 0.731 & Not rejected & 0.344 & Not rejected \\
\hline & $19: 00-20: 00$ & 35 & 0.436 & Not rejected & 0.839 & Not rejected \\
\hline & $20: 00-21: 00$ & 44 & 0.904 & Not rejected & 0.794 & Not rejected \\
\hline & $21: 00-22: 00$ & 43 & 0.459 & Not rejected & 0.693 & Not rejected \\
\hline & $22: 00-23: 00$ & 32 & 0.967 & Not rejected & 0.667 & Not rejected \\
\hline & $23: 00-24: 00$ & 31 & 0.306 & Not rejected & 0.552 & Not rejected \\
\hline
\end{tabular}


Table 5 (continued)

\begin{tabular}{|c|c|c|c|c|c|c|}
\hline \multirow[t]{2}{*}{$m$} & \multirow[t]{2}{*}{ Interval } & \multirow[t]{2}{*}{$k_{i}$} & \multicolumn{2}{|c|}{ CU KS test } & \multicolumn{2}{|c|}{ Dispersion test } \\
\hline & & & $p$-value & $H_{0}$ & $p$-value & $H_{0}$ \\
\hline & $00: 00-01: 00$ & 33 & 0.106 & Not rejected & 0.527 & Not rejected \\
\hline & $01: 00-02: 00$ & 22 & 0.658 & Not rejected & 0.488 & Not rejected \\
\hline & $02: 00-03: 00$ & 13 & 0.031 & Rejected & 0.390 & Not rejected \\
\hline & 03:00 - 04:00 & 14 & 0.258 & Not rejected & 0.857 & Not rejected \\
\hline & $04: 00-05: 00$ & 16 & 0.441 & Not rejected & 0.471 & Not rejected \\
\hline & $05: 00-06: 00$ & 22 & 0.707 & Not rejected & 0.335 & Not rejected \\
\hline & $06: 00-07: 00$ & 23 & 0.580 & Not rejected & 0.608 & Not rejected \\
\hline & 07:00 - 08:00 & 48 & 0.500 & Not rejected & 0.484 & Not rejected \\
\hline & 08:00 - 09:00 & 54 & 0.338 & Not rejected & 0.573 & Not rejected \\
\hline & 09:00 - 10:00 & 97 & 0.391 & Not rejected & 0.886 & Not rejected \\
\hline & $10: 00-11: 00$ & 97 & 0.149 & Not rejected & 0.836 & Not rejected \\
\hline & $11: 00-12: 00$ & 108 & 0.384 & Not rejected & 0.999 & Not rejected \\
\hline & $12: 00-13: 00$ & 95 & 0.911 & Not rejected & 0.821 & Not rejected \\
\hline & $13: 00-14: 00$ & 82 & 0.733 & Not rejected & 0.923 & Not rejected \\
\hline & $14: 00-15: 00$ & 75 & 0.979 & Not rejected & 0.753 & Not rejected \\
\hline & $15: 00-16: 00$ & 89 & 0.909 & Not rejected & 0.456 & Not rejected \\
\hline & $16: 00-17: 00$ & 82 & 0.429 & Not rejected & 0.923 & Not rejected \\
\hline & $17: 00-18: 00$ & 78 & 0.804 & Not rejected & 0.596 & Not rejected \\
\hline & $18: 00-19: 00$ & 69 & 0.277 & Not rejected & 0.734 & Not rejected \\
\hline & $19: 00-20: 00$ & 69 & 0.218 & Not rejected & 0.477 & Not rejected \\
\hline & $20: 00-21: 00$ & 72 & 0.731 & Not rejected & 0.731 & Not rejected \\
\hline & $21: 00-22: 00$ & 75 & 0.449 & Not rejected & 0.541 & Not rejected \\
\hline & $22: 00-23: 00$ & 60 & 0.989 & Not rejected & 0.681 & Not rejected \\
\hline & $23: 00-24: 00$ & 48 & 0.521 & Not rejected & 0.689 & Not rejected \\
\hline
\end{tabular}


Table 5 (continued)

\begin{tabular}{|c|c|c|c|c|c|c|}
\hline \multirow[t]{2}{*}{$m$} & \multirow[t]{2}{*}{ Interval } & \multirow[t]{2}{*}{$k_{i}$} & \multicolumn{2}{|c|}{ CU KS test } & \multicolumn{2}{|c|}{ Dispersion test } \\
\hline & & & $p$-value & $H_{0}$ & $p$-value & $H_{0}$ \\
\hline \multirow[t]{24}{*}{17} & 00:00 - 01:00 & 73 & 0.729 & Not rejected & 0.472 & Not rejected \\
\hline & $01: 00-02: 00$ & 48 & 0.708 & Not rejected & 0.291 & Not rejected \\
\hline & $02: 00-03: 00$ & 39 & 0.009 & Rejected & 0.010 & Rejected \\
\hline & 03:00 - 04:00 & 32 & 0.203 & Not rejected & 0.622 & Not rejected \\
\hline & $04: 00-05: 00$ & 28 & 0.706 & Not rejected & 0.652 & Not rejected \\
\hline & 05:00 - 06:00 & 38 & 0.125 & Not rejected & 0.607 & Not rejected \\
\hline & $06: 00-07: 00$ & 35 & 0.908 & Not rejected & 0.327 & Not rejected \\
\hline & 07:00 - 08:00 & 70 & 0.788 & Not rejected & 0.075 & Not rejected \\
\hline & 08:00 - 09:00 & 121 & 0.786 & Not rejected & 0.577 & Not rejected \\
\hline & $09: 00-10: 00$ & 174 & 0.421 & Not rejected & 0.729 & Not rejected \\
\hline & $10: 00-11: 00$ & 186 & 0.332 & Not rejected & 0.939 & Not rejected \\
\hline & $11: 00-12: 00$ & 203 & 0.474 & Not rejected & 0.999 & Not rejected \\
\hline & $12: 00-13: 00$ & 176 & 0.698 & Not rejected & 0.986 & Not rejected \\
\hline & $13: 00-14: 00$ & 164 & 0.589 & Not rejected & 0.992 & Not rejected \\
\hline & $14: 00-15: 00$ & 161 & 0.983 & Not rejected & 0.570 & Not rejected \\
\hline & $15: 00-16: 00$ & 168 & 0.506 & Not rejected & 0.815 & Not rejected \\
\hline & $16: 00-17: 00$ & 153 & 0.361 & Not rejected & 0.996 & Not rejected \\
\hline & $17: 00-18: 00$ & 149 & 0.596 & Not rejected & 0.528 & Not rejected \\
\hline & $18: 00-19: 00$ & 134 & 0.761 & Not rejected & 0.909 & Not rejected \\
\hline & $19: 00-20: 00$ & 140 & 0.101 & Not rejected & 0.637 & Not rejected \\
\hline & $20: 00-21: 00$ & 141 & 0.709 & Not rejected & 0.760 & Not rejected \\
\hline & $21: 00-22: 00$ & 153 & 0.938 & Not rejected & 0.855 & Not rejected \\
\hline & $22: 00-23: 00$ & 129 & 0.887 & Not rejected & 0.393 & Not rejected \\
\hline & $23: 00-24: 00$ & 94 & 0.950 & Not rejected & 0.296 & Not rejected \\
\hline
\end{tabular}


Table 5 (continued)

\begin{tabular}{|c|c|c|c|c|c|c|}
\hline \multirow[t]{2}{*}{$m$} & \multirow[t]{2}{*}{ Interval } & \multirow[t]{2}{*}{$k_{i}$} & \multicolumn{2}{|c|}{ CU KS test } & \multicolumn{2}{|c|}{ Dispersion test } \\
\hline & & & $p$-value & $H_{0}$ & $p$-value & $H_{0}$ \\
\hline \multirow[t]{24}{*}{22} & $00: 00-01: 00$ & 95 & 0.509 & Not rejected & 0.720 & Not rejected \\
\hline & $01: 00-02: 00$ & 70 & 0.938 & Not rejected & 0.529 & Not rejected \\
\hline & $02: 00-03: 00$ & 52 & 0.008 & Rejected & 0.022 & Rejected \\
\hline & $03: 00-04: 00$ & 36 & 0.094 & Not rejected & 0.507 & Not rejected \\
\hline & $04: 00-05: 00$ & 34 & 0.536 & Not rejected & 0.420 & not rejected \\
\hline & $05: 00-06: 00$ & 46 & 0.045 & Rejected & 0.703 & Not rejected \\
\hline & $06: 00-07: 00$ & 48 & 0.833 & Not rejected & 0.590 & Not rejected \\
\hline & $07: 00-08: 00$ & 83 & 0.805 & Not rejected & 0.062 & Not rejected \\
\hline & 08:00 - 09:00 & 165 & 0.576 & Not rejected & 0.108 & Not rejected \\
\hline & $09: 00-10: 00$ & 219 & 0.105 & Not rejected & 0.737 & Not rejected \\
\hline & $10: 00-11: 00$ & 235 & 0.282 & Not rejected & 0.960 & Not rejected \\
\hline & $11: 00-12: 00$ & 274 & 0.585 & Not rejected & 0.962 & Not rejected \\
\hline & $12: 00-13: 00$ & 233 & 0.956 & Not rejected & 0.984 & Not rejected \\
\hline & $13: 00-14: 00$ & 216 & 0.515 & Not rejected & 0.999 & Not rejected \\
\hline & $14: 00-15: 00$ & 207 & 0.872 & Not rejected & 0.789 & Not rejected \\
\hline & $15: 00-16: 00$ & 213 & 0.841 & Not rejected & 0.905 & Not rejected \\
\hline & $16: 00-17: 00$ & 204 & 0.491 & Not rejected & 0.999 & Not rejected \\
\hline & $17: 00-18: 00$ & 192 & 0.534 & Not rejected & 0.683 & Not rejected \\
\hline & $18: 00-19: 00$ & 173 & 0.818 & Not rejected & 0.968 & Not rejected \\
\hline & $19: 00-20: 00$ & 177 & 0.072 & Not rejected & 0.768 & Not rejected \\
\hline & $20: 00-21: 00$ & 181 & 0.655 & Not rejected & 0.681 & Not rejected \\
\hline & $21: 00-22: 00$ & 196 & 0.977 & Not rejected & 0.810 & Not rejected \\
\hline & $22: 00-23: 00$ & 167 & 0.688 & Not rejected & 0.412 & Not rejected \\
\hline & $23: 00-24: 00$ & 118 & 0.963 & Not rejected & 0.209 & Not rejected \\
\hline
\end{tabular}


Table 5 (continued)

\begin{tabular}{|c|c|c|c|c|c|c|}
\hline \multirow[t]{2}{*}{$m$} & \multirow[t]{2}{*}{ Interval } & \multirow[t]{2}{*}{$k_{i}$} & \multicolumn{2}{|c|}{ CU KS test } & \multicolumn{2}{|c|}{ Dispersion test } \\
\hline & & & $p$-value & $H_{0}$ & $p$-value & $H_{0}$ \\
\hline \multirow[t]{24}{*}{26} & $00: 00-01: 00$ & 112 & 0.171 & Not rejected & 0.679 & Not rejected \\
\hline & $01: 00-02: 00$ & 75 & 0.933 & Not rejected & 0.377 & Not rejected \\
\hline & $02: 00-03: 00$ & 67 & 0.012 & Rejected & 0.053 & Not rejected \\
\hline & 03:00 - 04:00 & 46 & 0.458 & Not rejected & 0.450 & Not rejected \\
\hline & $04: 00-05: 00$ & 38 & 0.987 & Not rejected & 0.465 & Not rejected \\
\hline & $05: 00-06: 00$ & 57 & 0.308 & Not rejected & 0.535 & Not rejected \\
\hline & $06: 00-07: 00$ & 56 & 0.935 & Not rejected & 0.739 & Not rejected \\
\hline & $07: 00-08: 00$ & 100 & 0.882 & Not rejected & 0.128 & Not rejected \\
\hline & 08:00 - 09:00 & 198 & 0.566 & Not rejected & 0.142 & Not rejected \\
\hline & 09:00 - 10:00 & 259 & 0.341 & Not rejected & 0.844 & Not rejected \\
\hline & $10: 00-11: 00$ & 289 & 0.091 & Not rejected & 0.942 & Not rejected \\
\hline & $11: 00-12: 00$ & 320 & 0.725 & Not rejected & 0.984 & Not rejected \\
\hline & $12: 00-13: 00$ & 274 & 0.915 & Not rejected & 0.996 & Not rejected \\
\hline & $13: 00-14: 00$ & 257 & 0.228 & Not rejected & 0.999 & Not rejected \\
\hline & $14: 00-15: 00$ & 243 & 0.872 & Not rejected & 0.835 & Not rejected \\
\hline & $15: 00-16: 00$ & 242 & 0.574 & Not rejected & 0.892 & Not rejected \\
\hline & $16: 00-17: 00$ & 236 & 0.630 & Not rejected & 0.942 & Not rejected \\
\hline & $17: 00-18: 00$ & 231 & 0.808 & Not rejected & 0.753 & Not rejected \\
\hline & $18: 00-19: 00$ & 204 & 0.682 & Not rejected & 0.980 & Not rejected \\
\hline & $19: 00-20: 00$ & 209 & 0.170 & Not rejected & 0.830 & Not rejected \\
\hline & $20: 00-21: 00$ & 219 & 0.610 & Not rejected & 0.735 & Not rejected \\
\hline & $21: 00-22: 00$ & 237 & 0.803 & Not rejected & 0.905 & Not rejected \\
\hline & $22: 00-23: 00$ & 198 & 0.614 & Not rejected & 0.366 & Not rejected \\
\hline & $23: 00-24: 00$ & 147 & 0.972 & Not rejected & 0.032 & Not rejected \\
\hline
\end{tabular}

From top to bottom: $m=5,9,17,22,26$. For each interval of each partition, the sample size of the dispersion test is $m . H_{0}$ denotes the null hypothesis of the corresponding test 
Table 6 Results of the CU KS and dispersion tests (with a significance level of 0.05) applied to each interval of the final (infeasible) partition obtained by solving problem 12 for different values of the parameter $m$, with $w$ fixed to 1

\begin{tabular}{|c|c|c|c|c|c|c|}
\hline \multirow[t]{2}{*}{$m$} & \multirow[t]{2}{*}{ Interval } & \multirow[t]{2}{*}{$k_{i}$} & \multicolumn{2}{|c|}{ CU KS test } & \multicolumn{2}{|c|}{ Dispersion test } \\
\hline & & & $p$-value & $H_{0}$ & $p$-value & $H_{0}$ \\
\hline \multirow[t]{10}{*}{5} & 00:00 - 06:00 & 65 & 0.068 & Not rejected & 0.472 & Not rejected \\
\hline & $06: 00-07: 00$ & 15 & 0.884 & Not rejected & 0.504 & Not rejected \\
\hline & $07: 00-08: 00$ & 27 & 0.820 & Not rejected & 0.164 & Not rejected \\
\hline & 08:00 - 09:00 & 35 & 0.875 & Not rejected & 0.534 & Not rejected \\
\hline & 09:00 - 10:00 & 50 & 0.378 & Not rejected & 0.844 & Not rejected \\
\hline & $10: 00-12: 00$ & 107 & 0.734 & Not rejected & 0.938 & Not rejected \\
\hline & $12: 00-13: 00$ & 51 & 0.594 & Not rejected & 0.765 & Not rejected \\
\hline & $13: 00-14: 00$ & 47 & 0.651 & Not rejected & 0.689 & Not rejected \\
\hline & $14: 00-15: 00$ & 44 & 0.817 & Not rejected & 0.412 & Not rejected \\
\hline & $15: 00-24: 00$ & 363 & 0.214 & Not rejected & 0.568 & Not rejected \\
\hline \multirow[t]{13}{*}{9} & $00: 00-02: 00$ & 55 & 0.249 & Not rejected & 0.607 & Not rejected \\
\hline & $02: 00-04: 00$ & 27 & 0.309 & Not rejected & 0.501 & Not rejected \\
\hline & $04: 00-05: 00$ & 16 & 0.441 & Not rejected & 0.471 & Not rejected \\
\hline & 05:00 - 06:00 & 22 & 0.707 & Not rejected & 0.335 & Not rejected \\
\hline & $06: 00-07: 00$ & 23 & 0.580 & Not rejected & 0.608 & Not rejected \\
\hline & 07:00 - 08:00 & 48 & 0.500 & Not rejected & 0.484 & Not rejected \\
\hline & 08:00 - 09:00 & 54 & 0.338 & Not rejected & 0.573 & Not rejected \\
\hline & $09: 00-16: 00$ & 643 & 0.060 & Not rejected & 0.717 & Not rejected \\
\hline & $16: 00-17: 00$ & 82 & 0.429 & Not rejected & 0.923 & Not rejected \\
\hline & $17: 00-18: 00$ & 78 & 0.804 & Not rejected & 0.596 & Not rejected \\
\hline & $18: 00-22: 00$ & 285 & 0.919 & Not rejected & 0.989 & Not rejected \\
\hline & $22: 00-23: 00$ & 60 & 0.989 & Not rejected & 0.681 & Not rejected \\
\hline & $23: 00-24: 00$ & 48 & 0.522 & Not rejected & 0.689 & Not rejected \\
\hline \multirow[t]{12}{*}{17} & $00: 00-02: 00$ & 121 & 0.094 & Not rejected & 0.535 & Not rejected \\
\hline & $02: 00-05: 00$ & 99 & 0.098 & Not rejected & 0.067 & Not rejected \\
\hline & $05: 00-07: 00$ & 73 & 0.650 & Not rejected & 0.203 & Not rejected \\
\hline & 07:00 - 08:00 & 70 & 0.788 & Not rejected & 0.075 & Not rejected \\
\hline & 08:00 - 09:00 & 121 & 0.786 & Not rejected & 0.577 & Not rejected \\
\hline & $09: 00-10: 00$ & 174 & 0.421 & Not rejected & 0.729 & Not rejected \\
\hline & $10: 00-14: 00$ & 729 & 0.089 & Not rejected & 0.995 & Not rejected \\
\hline & $14: 00-16: 00$ & 329 & 0.982 & Not rejected & 0.410 & Not rejected \\
\hline & $16: 00-17: 00$ & 153 & 0.361 & Not rejected & 0.996 & Not rejected \\
\hline & $17: 00-18: 00$ & 149 & 0.596 & Not rejected & 0.528 & Not rejected \\
\hline & $18: 00-22: 00$ & 568 & 0.586 & Not rejected & 0.926 & Not rejected \\
\hline & $22: 00-24: 00$ & 223 & 0.071 & Not rejected & 0.793 & Not rejected \\
\hline
\end{tabular}


Table 6 (continued)

\begin{tabular}{|c|c|c|c|c|c|c|}
\hline \multirow[t]{2}{*}{$m$} & \multirow[t]{2}{*}{ Interval } & \multirow[t]{2}{*}{$k_{i}$} & \multicolumn{2}{|c|}{ CU KS test } & \multicolumn{2}{|c|}{ Dispersion test } \\
\hline & & & $p$-value & $H_{0}$ & $p$-value & $H_{0}$ \\
\hline \multirow[t]{13}{*}{22} & $00: 00-02: 00$ & 165 & 0.198 & Not rejected & 0.743 & Not rejected \\
\hline & $02: 00-06: 00$ & 168 & 0.117 & Not rejected & 0.122 & Not rejected \\
\hline & $06: 00-07: 00$ & 48 & 0.833 & Not rejected & 0.590 & Not rejected \\
\hline & 07:00 - 08:00 & 83 & 0.805 & Not rejected & 0.062 & Not rejected \\
\hline & 08:00 - 09:00 & 165 & 0.576 & Not rejected & 0.108 & Not rejected \\
\hline & 09:00 - 10:00 & 219 & 0.105 & Not rejected & 0.737 & Not rejected \\
\hline & $10: 00-14: 00$ & 958 & 0.097 & Not rejected & 0.994 & Not rejected \\
\hline & $14: 00-16: 00$ & 420 & 0.952 & Not rejected & 0.561 & Not rejected \\
\hline & $16: 00-17: 00$ & 204 & 0.491 & Not rejected & 0.999 & Not rejected \\
\hline & $17: 00-18: 00$ & 192 & 0.534 & Not rejected & 0.683 & Not rejected \\
\hline & $18: 00-22: 00$ & 772 & 0.436 & Not rejected & 0.968 & Not rejected \\
\hline & $22: 00-23: 00$ & 167 & 0.688 & Not rejected & 0.412 & Not rejected \\
\hline & $23: 00-24: 00$ & 118 & 0.963 & Not rejected & 0.209 & Not rejected \\
\hline \multirow[t]{16}{*}{26} & $00: 00-01: 00$ & 112 & 0.171 & Not rejected & 0.679 & Not rejected \\
\hline & 01:00 - 02:00 & 75 & 0.933 & Not rejected & 0.378 & Not rejected \\
\hline & 02:00 - 06:00 & 208 & 0.072 & Not rejected & 0.080 & Not rejected \\
\hline & $06: 00-07: 00$ & 56 & 0.935 & Not rejected & 0.739 & Not rejected \\
\hline & 07:00 - 08:00 & 100 & 0.882 & Not rejected & 0.128 & Not rejected \\
\hline & 08:00 - 09:00 & 198 & 0.566 & Not rejected & 0.142 & Not rejected \\
\hline & 09:00 - 10:00 & 259 & 0.341 & Not rejected & 0.844 & Not rejected \\
\hline & $10: 00-11: 00$ & 289 & 0.091 & Not rejected & 0.942 & Not rejected \\
\hline & $11: 00-12: 00$ & 320 & 0.725 & Not rejected & 0.984 & Not rejected \\
\hline & $12: 00-13: 00$ & 274 & 0.915 & Not rejected & 0.996 & Not rejected \\
\hline & $13: 00-15: 00$ & 500 & 0.439 & Not rejected & 0.971 & Not rejected \\
\hline & $15: 00-16: 00$ & 242 & 0.574 & Not rejected & 0.892 & Not rejected \\
\hline & $16: 00-18: 00$ & 467 & 0.895 & Not rejected & 0.939 & Not rejected \\
\hline & $18: 00-21: 00$ & 632 & 0.643 & Not rejected & 0.950 & Not rejected \\
\hline & $21: 00-22: 00$ & 237 & 0.803 & Not rejected & 0.905 & Not rejected \\
\hline & $22: 00-24: 00$ & 345 & 0.034 & Rejected & 0.440 & Not rejected \\
\hline
\end{tabular}

From top to bottom: $m=5,9,17,22,26$. For each interval of each partition, the sample size of the dispersion test is $m . H_{0}$ denotes the null hypothesis of the corresponding test

Funding Open access funding provided by Università degli Studi di Roma La Sapienza within the CRUICARE Agreement.

\section{Declarations}

Conflict of interest The authors declare that they have no conflict of interest. 
Open Access This article is licensed under a Creative Commons Attribution 4.0 International License, which permits use, sharing, adaptation, distribution and reproduction in any medium or format, as long as you give appropriate credit to the original author(s) and the source, provide a link to the Creative Commons licence, and indicate if changes were made. The images or other third party material in this article are included in the article's Creative Commons licence, unless indicated otherwise in a credit line to the material. If material is not included in the article's Creative Commons licence and your intended use is not permitted by statutory regulation or exceeds the permitted use, you will need to obtain permission directly from the copyright holder. To view a copy of this licence, visit http://creativecommons.org/licen ses/by/4.0/.

\section{References}

Ahalt V, Argon N, Strickler J, Mehrotra A (2018) Comparison of emergency department crowding scores: a discrete-event simulation approach. Health Care Manag Sci 21:144-155

Ahmed MA, Alkhamis TM (2009) Simulation optimization for an emergency department healthcare unit in Kuwait. Eur J Oper Res 198(3):936-942

Audet C, Hare W (2017) Derivative-free and blackbox optimization. Springer Series in Operations Research and Financial Engineering, Springer

Bernstein S, Verghese V, Leung W, Lunney TA, Perez I (2003) Development and validation of a new index to measure emergency department crowding. Acad Emerg Med 10:938-42

Brown L, Gans N, Mandelbaum A, Sakov A, Shen H, Zeltyn S, Zhao L (2005) Statistical analysis of a telephone call center: a queueing-science perspective. J Am Stat Assoc 100:36-50

Conn A, Scheinberg K, Vicente L (2009) Derivative-Free Optimization. SIAM

Daldoul D, Nouaouri I, Bouchriha H, Allaoui H (2018) A stochastic model to minimize patient waiting time in an emergency department. Oper Res Health Care 18:16-25, EURO 2016-New Advances in Health Care Applications

De Santis A, Giovannelli T, Lucidi S, Messedaglia M, Roma M (2020) An optimal non-uniform piecewise constant approximation for the patient arrival rate for a more efficient representation of the Emergency Departments arrival process. Technical Report 1-2020, Dipartimento di Ingegneria Informatica Automatica e Gestionale “A. Ruberti”, SAPIENZA Università di Roma

Durbin J (1961) Some methods for constructing exact tests. Biometrika 48:41-55

Gartner D, Padman R (2020) Machine learning for healthcare behavioural OR: addressing waiting time perceptions in emergency care. J Oper Res Soc 71:1087-1101

Guo H, Goldsman D, Tsui KL, Zhou Y, Wong SY (2016) Using simulation and optimisation to characterise durations of emergency department service times with incomplete data. Int $\mathbf{J}$ Prod Res 54(21):6494-6511

Guo H, Gao S, Tsui K, Niu T (2017) Simulation optimization for medical staff configuration at emergency department in Hong Kong. IEEE Trans Autom Sci Eng 14(4):1655-1665

Hoot N, Aronsky D (2008) Systematic review of emergency department crowding: causes, effects, and solutions. Ann Emerg Med 52(2):126-136

Hoot NR, Zhou CH, Jones I, Aronsky D (2007) Measuring and forecasting emergency department crowding in real time. Ann Emerg Med 49(6):747-55

Reeder JT, Burleson DG, Garrison H (2003) The overcrowded emergency department: a comparison of staff perceptions. Acad Emerg Med 10:1059-64

Kathirgamatamby N (1953) Note on the Poisson index of dispersion. Biometrika 40:225-228

Kim SH, Whitt W (2014a) Are call center and hospital arrivals well modeled by nonhomogeneous Poisson process?? Manuf Serv Oper Manag 16:464-480

Kim SH, Whitt W (2014b) Choosing arrival process models for service systems: tests of a nonhomogeneous Poisson process. Nav Res Logist 61:66-90

Kim SH, Whitt W (2015) The power of alternative Kolmogorov-Smirnov tests based on transformations of the data. ACM Trans Model Comput Simul 25(4):1-22

Kuo YH, Rado O, Lupia B, Leung JMY, Graham CA (2016) Improving the efficiency of a hospital emergency department: a simulation study with indirectly imputed service-time distributions. Flex Serv Manuf J 28(1):120-147

Larson J, Menickelly M, Wild S (2019) Derivative-free optimization methods. Acta Numer 28:287-404 
Lewis P (1965) Some results on tests for Poisson processes. Biometrika 52:67-77

Liuzzi G, Lucidi S, Rinaldi F (2020) An algorithmic framework based on primitive directions and nonmonotone line searches for black-box problems with integer variables, Mathematical Programming Computation

Salmon A, Rachuba S, Briscoe S, Pitt M (2018) A structured literature review of simulation modelling applied to Emergency Departments: current patterns and emerging trends. Oper Res Health Care 19:1-13

Ucar I, Smeets B, Azcorra A (2019) Simmer: discrete-event simulation for R. J Stat Softw 90(2):1-30

Vanbrabant L, Braekers K, Ramaekers K (2020) Improving emergency department performance by revising the patient-physician assignment process. Flex Serv Manuf J

Vile J, Gillard J, Harper P, Knight V (2017) A queueing theoretic approach to set staffing levels in time dependent dual class service systems. Decis Sci 48:766-794

Wang H, Robinson RD, Garrett JS, Bunch K, Huggins CA, Watson K, Daniels J, Banks B, D'Etienne JP, Zenarosa NR (2015) Use of the SONET score to evaluate high volume emergency department overcrowding: a prospective derivation and validation study. Emerg Med Int 11:1-11

Weiss S, Derlet R, Arndahl J, Ernst A, Richards J, Fernández-Frankelton M, Schwab R, Stair T, Vicellio P, Levy D, Brautigan M, Johnson A, Nick T (2004) Estimating the degree of emergency department overcrowding in academic medical centers: results of the national ed overcrowding study (NEDOCS). Acad Emerg Med 11(1):38-50

Weiss S, Ernst AA, Nick TG (2006) Comparison of the national emergency department overcrowding scale and the emergency department work index for quantifying emergency department crowding. Acad Emerg Med 13(5):513-8

Wiler J, Griffey R, Olsen T (2011) Review of modeling approaches for emergency department patient flow and crowding research. Acad Emerg Med 18:1371-1379

Zeinali F, Mahootchi M, Sepehri M (2015) Resource planning in the emergency departments: a simulation-base metamodeling approach. Simul Model Pract Theory 53:123-138

Publisher's Note Springer Nature remains neutral with regard to jurisdictional claims in published maps and institutional affiliations.

\begin{abstract}
Alberto De Santis is Associate Professor at the Department of Computer, Control and Management Engineering, Sapienza University of Rome, Rome, Italy. His research activity has been mainly in two fields. Modelling, identification, and filtering of linear systems: in this framework he worked on signal and image processing, with applications in industrial as well as in the biomedical fields. Filtering and control theory: he studied problems related to optimal filtering and identification of systems affected by white noise; the robust stabilization stochastic nonlinear systems.
\end{abstract}

Tommaso Giovannelli is a Ph.D. student in Operations Research in the Department of Computer, Control and Management Engineering at Sapienza University of Rome, Rome, Italy, where he received an M.S. in management engineering in 2017. Since 2020 he has been a visiting research scholar in the Department of Industrial and Systems Engineering at Lehigh University, Bethlehem, PA. His current research interests include nonlinear optimization, derivative-free optimization, simulation-based optimization, and operations research applied to healthcare management.

Stefano Lucidi is Full Professor at Sapienza University of Rome, Rome, Italy. He teaches Operations Research in the first-level degrees in management engineering and Continuous Optimization in the second-level degrees. His research interests are mainly focused on the study and definition of nonlinear optimization methods and their applications in several different fields such as machine learning, optimal design, management of healthcare services, analysis of gene expressions, and planning problems of cruise itineraries.

Mauro Messedaglia is received the M.S. degree in aeronautical engineering from the Sapienza University of Rome, Rome, Italy, in 1992. He was a Software Engineer, Project Manager, and Data Scientist for IT Company from 1994 to 2020. Since 2015 he has been a Consultant of ACTOR Start up of Sapienza University of Rome. His current research interests include simulation and optimization modeling, data mining, and text mining. 
Massimo Roma is received the M.S. degree in mathematics (summa cum laude) and the Ph.D. degree in Operations Research from the Sapienza University of Rome, Rome, Italy, in 1988 and 1995, respectively. He was an Assistant Professor with the Department of Computer, Control and Management Engineering Antonio Ruberti, Sapienza University of Rome, from 1996 to 2004, where he has been Associate Professor of Operations Research, since 2005. He currently teaches the course on Operations Research in the B.S. program in Engineering in Computer Science and Control Engineering, and the course on queueing theory and simulation in the M.S. program in Management Engineering with the Sapienza University of Rome. His current research interests include nonlinear optimization, simulation-based optimization, and the application of optimization and simulation methods to real-world problems.

\title{
Authors and Affiliations
}

\section{Alberto De Santis ${ }^{1}$ (D) Tommaso Giovannelli $^{1}$ (D) Stefano Lucidi ${ }^{1}$. Mauro Messedaglia ${ }^{2}$ - Massimo Roma ${ }^{1}$}

\author{
Alberto De Santis \\ desantis@diag.uniroma1.it \\ Stefano Lucidi \\ lucidi@diag.uniroma1.it \\ Mauro Messedaglia \\ mauro.messedaglia@gmail.com \\ Massimo Roma \\ roma@diag.uniroma1.it \\ 1 Dipartimento di Ingegneria Informatica, Automatica e Gestionale “A. Ruberti” SAPIENZA, \\ Università di Roma, via Ariosto, 25, 00185 Roma, Italy
}

2 ACTOR Start up of SAPIENZA Università di Roma, via Nizza 45, 00198 Roma, Italy 